\title{
Data report: electrical properties of gabbroic and troctolitic rocks from IODP Hole U1309D, Atlantis Massif ${ }^{1}$
}

\author{
Benoît Ildefonse, ${ }^{2}$ Marion Drouin, ${ }^{2}$ Marie Violay, ${ }^{2}$ and Philippe Pezard ${ }^{2}$
}

\section{Chapter contents}

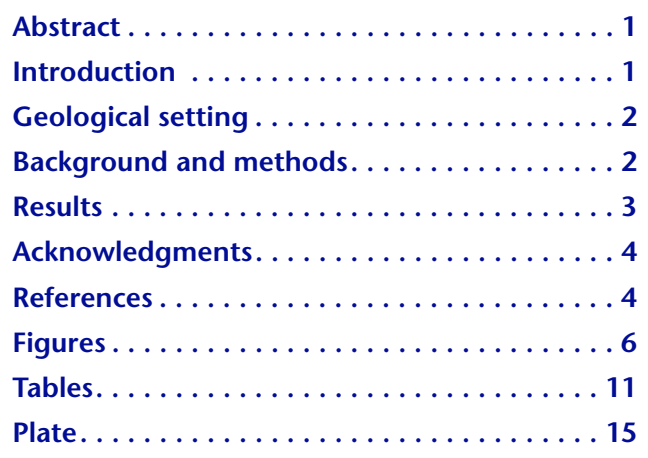

'1ldefonse, B., Drouin, M., Violay, M., and Pezard, P., 2009. Data report: electrical properties of gabbroic and troctolitic rocks from IODP Hole U1309D, Atlantis Massif. In Blackman, D.K., Ildefonse, B., John, B.E., Ohara, Y., Miller, D.J., MacLeod, C.J., and the Expedition 304/305 Scientists, Proc. IODP, 304/305: College Station, TX (Integrated Ocean Drilling Program Management International, Inc.).

doi:10.2204/iodp.proc.304305.204.2009

${ }^{2}$ Géosciences Montpellier, CNRS, Université Montpellier II, 34095 Montpellier Cedex 5, France. Correspondence author: benoit.ildefonse@univmontp2.fr

\begin{abstract}
In this report we present the results of laboratory measurements carried out to explore the electrical properties of gabbroic and troctolitic samples from Integrated Ocean Drilling Program (IODP) Hole U1309D in the Atlantis Massif, an oceanic core complex located at $30^{\circ} \mathrm{N}$ on the Mid-Atlantic Ridge. Oceanic core complexes are presumably localized in portions of heterogeneous oceanic crust that are relatively rich in igneous rocks and are the locus of significant hydrothermal activity. Electrical properties of igneous rocks are used to discuss the rocks' porosity structure as a function of mineralogy, alteration processes, and deformation. Electrical properties of the gabbroic suites sampled during IODP Expedition 304/305 can contribute to the understanding of these processes in the Atlantis Massif. We present the full suite of electrical data together with density and porosity measurements on the same suite of samples.
\end{abstract}

\section{Introduction}

One of the main objectives of Integrated Ocean Drilling Program (IODP) Expedition 304/305 was to drill a deep hole inside the Atlantis Massif, an oceanic core complex located at $30^{\circ} \mathrm{N}$ in the inside corner of the intersection of the Mid-Atlantic Ridge with the Atlantis Fracture Zone (see the "Expedition 304/305 summary" chapter). IODP Hole U1309D was drilled to 1415.5 meters below seafloor (mbsf); it is the second deepest hole in slow-spread crust after Ocean Drilling Program (ODP) Hole 735B on the Southwest Indian Ridge (Dick et al., 2000). In this report we present a series of laboratory measurements carried out at Géosciences Montpellier (France) to quantify the electrical properties of the oceanic crustal rocks recovered from Hole U1309D. Our knowledge of the in situ structure of oceanic crust is partly based on geophysical investigations, including downhole measurements (e.g., Goldberg, 1997). The analysis of marine geophysical data requires a complete understanding of the intrinsic physical properties of investigated crustal sections. Electrical measurements are used to evaluate the porosity structure of low-porosity igneous rocks (e.g., Pezard, 1990) and are presented in this report together with density and porosity measurements. This work follows the study of electrical properties of gabbroic rocks sampled in Hole 735B at the 
Southwest Indian Ridge (Pezard et al., 1991; Ildefonse and Pezard, 2001)

\section{Geological setting}

Hole U1309D is almost exclusively composed of gabbroic and troctolitic rocks, with the exception of a few tens of centimeters of mantle peridotites in the upper $225 \mathrm{~m}$ (see the "Site U1309" chapter; Ildefonse et al., 2006). In comparison with gabbroic sections previously recovered by drilling in oceanic crust (Robinson, Von Herzen, et al., 1989; Gillis, Mével, Allan, et al., 1993; Cannat, Karson, Miller, et al., 1995; Dick, Natland, Miller, et al., 1999; Pettigrew, Casey, Miller, et al., 1999; Kelemen, Kikawa, Miller, et al., 2004), the core recovered from Hole U1309D comprises a larger proportion of olivinerich lithologies. On the basis of their modal abundance in olivine, plagioclase, clinopyroxene, and FeTi oxide, gabbroic rocks were grouped after shipboard descriptions (see the "Site U1309" chapter) into olivine-rich troctolite (5.4\% of recovered rocks), troctolite $(2.7 \%)$, olivine to troctolitic gabbro $(28 \%)$, gabbro (56\%), and oxide gabbro (7\%). Olivine-rich troctolites have more than $\sim 70 \%$ olivine as subhedral or rounded grains included in interstitial to poikilitic plagioclase and clinopyroxene. Their modal compositions vary at a scale of a few centimeters to a few decimeters, locally grading to dunite, wehrlite, or olivine gabbro. Olivine gabbro ( $>5 \%$ olivine) and gabbro display seriated textures and are characterized by significant variations in grain size and modal composition on a decimeter scale. Olivine gabbro locally grades to troctolitic gabbro and troctolite. Gabbro is generally intrusive into troctolite and olivine gabbro, with either sharp or locally more diffuse contacts. Thin section observation reveals the occurrence of significant amounts of orthopyroxene in gabbros below 600 mbsf. Oxide gabbros ( $>2 \%$ modal Fe-Ti oxides) occur as disseminated patches, dikelets crosscutting other lithologies, or, less often, associated with ductile deformation zones (see the "Site U1309" chapter). Late-stage dikes of coarse-grained gabbro and microgabbro commonly intrude troctolite units. The core is variably altered at conditions ranging from granulite to zeolite facies. Overall, alteration tends to decrease downhole, and olivine-rich troctolite intervals between 1090 and 1230 mbsf are locally exceptionally fresh. Magmatic deformation associated with the emplacement of the Hole U1309D gabbroic intrusive suite is weak and affects only $22 \%$ of the recovered core. High-temperature (amphibolite facies) deformation is rare, and high-strain ductile shear zones represent $<3 \%$ of the recovered core.

Hole U1309D is the last of 16 holes drilled in four different oceanic core complexes in the Atlantic and Indian Oceans (ODP Legs 118, 153, 176, 179, and 209 and Expedition 304/305), all of which recovered only gabbroic sections. This common geological feature in boreholes, contrasting with the more variable seafloor geology in the same areas, including serpentinites, was the basis for a revised model of oceanic core complex development, in which the core is dominantly composed of gabbro intrusion(s) surrounded by faults preferentially localized in serpentinized peridotites (Ildefonse et al., 2007). The detachment fault that caps the Atlantis Massif and other oceanic core complexes is the locus of abundant hydrothermal fluid circulation (McCaig et al., 2007). Characterizing the electrical properties of the gabbroic series recovered from Hole U1309D sheds light on the porosity structure, which is an important parameter, together with alteration petrology, for assessing the structure and dynamics of a hydrothermal system.

\section{Background and methods}

Electrical properties of porous media are sensitive to fluid content and to alteration and can thus be used to detect conductive features in a resistive matrix (e.g., Walsh and Brace, 1984; Pezard and Luthi, 1988). Modes of electrical conduction are identified in the laboratory from resistivity measurements at various saturating fluid salinities and provide information on the porosity structure and degree of alteration. The method was initially proposed for sandstones and clays (Waxman and Smits, 1968; Revil and Glover, 1998) but has been successfully applied to various low-porosity igneous rocks such as basalts (e.g., Pezard, 1990; Einaudi et al., 2000), gabbros (Pezard et al., 1991; Ildefonse and Pezard, 2001), peridotites (Ildefonse et al., 1999), and granites (Pape et al., 1985; Pezard et al., 1999; Belghoul, 2007).

In porous media composed of a matrix considered as infinitely resistive and a connected pore space saturated with a conductive electrolyte, two main mechanisms are responsible for electrical conduction: (1) an electrolytic conduction mechanism in pore volumes and (2) a surface conduction mechanism at the interface between the electrolyte and minerals. Electrolytic conduction is related to the nature and salinity of the saturating fluid; surface conduction is related to the presence of charges along pore surfaces, 
which is related at the first order to alteration (e.g., Revil and Glover, 1998). The measured electrical conductivity can be written as follows (Waxman and Smits, 1968):

$$
C_{0}=\left(C_{\mathrm{W}} / F\right)+C_{\mathrm{S}}
$$

where

$C_{0}=$ total conductivity of the pore space,

$C_{\mathrm{W}}=$ conductivity of water (saturating fluid),

$F=$ electrical formation factor, and

$C_{\mathrm{S}}=$ surface conductivity.

$C_{\mathrm{S}}$ is related to claylike silicates, hence to alteration through the cation exchange capacity (Waxman and Smits, 1968). This model works well at high salinity; however, it tends to overestimate $C_{\mathrm{s}}$ at low salinity. Alternatively, we can use the nonempirical statistical approach proposed by Revil and Glover (1998) based on the microgeometry of the porous space. The conductivity of the sample is then given by a complex model that has two simpler forms at high and low salinities (see equations in Revil and Glover, 1998; Ildefonse and Pezard, 2001).

To characterize their electrolytic and surface conduction components, each sample (minicore) was analyzed by measuring electrical resistivity at $1 \mathrm{kHz}$ and variable saturating fluid salinity (six series of measurements, from 0.06 to $60 \mathrm{~g} / \mathrm{L}$ ) (Fig. F1; Tables T1, T2). Measurements were taken with two electrodes; the polarizing effect at the electrode/sample interface was reduced by using paper filter to separate the electrodes from the sample (see Pezard, 1990, for a detailed description of the experimental protocol).

The contribution of surface conduction to the total measured electrical conductivity can be estimated by using $\beta$ (Ildefonse and Pezard, 2001):

$$
\beta=\left(F \times C_{\mathrm{s}}\right) /\left[\left(F \times C_{\mathrm{s}}\right)+C_{\mathrm{W}}\right],
$$

where $C_{\mathrm{W}}$ is $5000 \mathrm{mS} / \mathrm{m}$ (for seawater at $24^{\circ} \mathrm{C}$ ).

The intrinsic $F$ and the surface conductivity are extracted from the high- and low-salinity parts of the curves, respectively, using both the Waxman and Smits (1968) and Revil and Glover (1998) models.

$F$ depends only on the microstructural characteristics of the rock and is classically considered to characterize the three-dimensional topology of the pore space (e.g., Guéguen and Palciauskas, 1992). An empirical relationship between $F$ and porosity $(\phi)$ was proposed by Archie (1942) with:

$$
F=\phi^{-\mathrm{m}} .
$$

In the oil industry the exponent $m$ is called the "cementation index." The exponent $m$ typically varies from 1.5 to 2.5 in crystalline rocks (e.g., Guéguen and Palciauskas, 1992).

The relation between $F$ and $\phi$ can also be expressed in terms of degree of connectivity of the inner pore space, characterized by electrical the tortuosity $(\tau)$ (Walsh and Brace, 1984; Pezard, 1990; Pezard et al., 1991; Guéguen and Palciauskas, 1992):

$$
F=\tau / \phi
$$

Whereas $m$ describes the nonuniformity of the section of the conductive channels, $\tau$ relates to the complexity of the path followed by the electrical current (e.g., Guéguen and Palciauskas, 1992) or, in a more general sense, the efficiency of electrical flow processes (Clennell, 1997). In igneous low-porosity rocks, the average electrical tortuosity is generally on the order of 10 (e.g., Pezard et al., 1991; Ildefonse and Pezard, 2001).

Density and porosity were measured using the classic triple weighing method, with an OHAUS precision scale $\left(10^{-4} \mathrm{~g}\right.$ accuracy). Samples were first weighed after being dried in an oven at $\sim 50^{\circ} \mathrm{C}$ and resaturated for "wet" weight measurements in air and immersed. Bulk density $\left(\rho_{b}\right)$ and grain density $\left(\rho_{g}\right)$ are given by:

$$
\rho_{\mathrm{b}}=\left[M_{\text {sat }} /\left(M_{\text {sat }}-M_{\text {imm }}\right)\right] \times \rho_{\text {water }}
$$

and

$$
\rho_{\mathrm{g}}=\left[M_{\mathrm{dry}} /\left(M_{\mathrm{dry}}-M_{\mathrm{imm}}\right)\right] \times \rho_{\text {water }}
$$

where

$$
\begin{aligned}
& M_{\text {dry }}=\text { dry sample mass, } \\
& M_{\text {sat }}=\text { saturated sample mass, } \\
& M_{\text {imm }}=\text { the mass of the immersed saturated sam- } \\
& \text { ple, and } \\
& \rho_{\text {water }}=1.02 \mathrm{~g} / \mathrm{cm}^{3} \text { for a } 30 \mathrm{~g} / \mathrm{L} \text { salinity at } 0.1 \mathrm{MPa} \\
& \text { and } 20^{\circ}-25^{\circ} \mathrm{C} \text {. }
\end{aligned}
$$

The closed, unconnected porosity is supposed to be negligible; the connected porosity (in percent) is:

$$
\phi=100 \times\left[\left(M_{\text {sat }}-M_{\text {dry }}\right) /\left(M_{\text {sat }}-M_{\text {imm }}\right) .\right.
$$

\section{Results}

Measurements were obtained from 109 samples (Tables T1, T2, T3; Plate P1) taken from the entire core in Hole U1309D, which include 7 oxide gabbros (oxide content $>2 \%$ ), 72 gabbros and olivine gabbros, 14 troctolitic gabbros and troctolites, and 16 olivinerich troctolites (olivine $>\sim 70 \%$ ). See the "Site U1309" chapter for further descriptions of the lithologies recovered from Hole U1309D. 
Data quality is variable and was visually assessed on a case by case basis using an empirical scale for fit quality (from 1 = good to 3 = bad) (Fig. F1; Table T3). In 25 cases, the lowest salinity point tends to be low enough that it causes a deflection of the Revil and Glover (1998) model fit curve toward lower $C_{\mathrm{s}}$ values (Fig. F1C). This behavior was observed by Revil et al. (2002) in volcaniclastic material and was ascribed to the presence of zeolite in the samples. It is observed together with unusually high surface conductivities, which is also the case in our sample set. The 25 samples that show a deflection in the fit curve have high surface conductivities of $>2.5 \mathrm{mS} / \mathrm{m}$ (mean $=19.7$ $\mathrm{mS} / \mathrm{m}$ ), whereas the others have much lower surface conductivities of $<2.6 \mathrm{mS} / \mathrm{m}$ (mean $=0.57 \mathrm{mS} / \mathrm{m})$. Zeolite was described in Hole U1309D rocks (see the "Site U1309" chapter); hence, a similar behavior may be seen here. However, zeolite was only documented in the lower half of the hole, below $~ 700$ mbsf (see the "Site U1309" chapter), whereas this electrical behavior is observed in 14 samples evenly distributed above 700 mbsf. Assessing the potential role of zeolite in the measured samples requires characterizing their alteration mineralogy, which is beyond the objective of this data report. An alternative or complementary contributor to these seemingly anomalous results could be serpentinite and/or associated minerals (e.g., brucite, talc, or magnetite), as many of these samples have a primary olivine-rich composition. We could not, however, identify a simple relationship between this peculiar electrical behavior and a compositional or textural parameter such as the amount of serpentinite (several heavily serpentinized samples do not show this behavior) or fracture density. One additional complexity to the problem of understanding this behavior is that in several cases, the low-salinity deflection of the Revil and Glover (1998) model fit curve is not observed, whereas the lowest salinity measurement clearly lies below the fit curve (e.g., Fig. F1B). Some poor-quality measurements in the higher salinity range, for example, may prevent the model from properly fitting the whole data set. When removing these samples from the data set, the remaining samples that show the standard curve fit as shown in Figure F1A correspond to samples with lowest degrees $(<10 \%)$ of serpentinization.

In 21 samples, one or two points in the series of conductivity measurements were removed from the analyses because they were several orders of magnitude out of range when compared to the other points (Fig. F1C). In the majority of these 21 cases, the "bad" points were the lowest salinity measurements, as illustrated in Figure F1C, and we cannot exclude that these correspond to end-member ver- sions of the zeolite-like behavior described above. Except for one oxide gabbro, these contain significant amounts of more or less altered olivine (olivine gabbro to olivine-rich troctolite).

The standard errors of $F$ and $C_{\mathrm{s}}$ obtained from the analysis detailed above are given in Table $\mathrm{T} 2$ and shown in downhole distributions of $F$ and $C_{\mathrm{S}}$ for both the Waxman and Smits (1968) and Revil and Glover (1998) models (Fig. F2). In most cases, the largest errors are smaller than the small-scale trends observed in these distributions, which then must correspond to real changes in some first-order controlling parameter(s) such as porosity or alteration. Downhole distributions of electrical properties are also shown in Figure F3, together with porosity and density and compared to the downhole distribution of lithology, variation of hand sample alteration, and borehole electrical resistivity.

As classically done in studies of electrical properties, the measurements are shown as a function of sample porosity, $\phi$ (Figure F4). Correlations in gabbros (Ildefonse and Pezard, 2001; Einaudi et al., 2005) and granites (Belghoul, 2007), such as a linear decrease of $F$ and increases in $C_{S}, \tau$, and $m$ with $\phi$, are still present but somehow disturbed by samples that tend to have higher $C_{S}$ and lower $F$ at fairly constant $\phi$ $(\sim 1 \%)$. As a result, $\tau$ and $m$ tend to be more scattered, especially when abundant olivine/serpentine is present. Figure F5 shows the variations of $\phi, \tau$, and $m$ as a function of the degree of fracturation (intensity of cataclastic fabric, as characterized onboard on hand samples) and the degree of serpentinization. Note that higher degrees of serpentinization do not significantly change porosity and tend to create a simpler geometry of the porous network (slightly lower $\tau$ and $m$ ). Not surprisingly, $\phi, \tau$, and $m$ tend to increase in more fractured rocks.

\section{Acknowledgments}

This research used samples and/or data provided by the Integrated Ocean Drilling Program (IODP). Funding for this research was provided by the Centre Nationale de Recherche Scientifique-Institut National des Sciences de l'Univers Dynamique et Evolution de la Terre Interne (DyETI) program. We are grateful to Christophe Nevado and Doriane Delmas for providing high-quality thin sections.

\section{References}

Archie, G.E., 1942. The electrical resistivity log as an aid in determining some reservoir characteristics. J. Pet. Technol., 5:1-8. 
Belghoul, A., 2007. Caractérisation petrophysique et hydrodynamique du socle cristallin [Thèse de doctorat]. Université Montpellier 2.

Cannat, M., Karson, J.A., Miller, D.J., et al., 1995. Proc. ODP, Init. Repts., 153: College Station, TX (Ocean Drilling Program). doi:10.2973/odp.proc.ir.153.1995

Clennell, M.B., 1997. Tortuosity: a guide through the maze. In Lovell, M.A., and Harvey, P.K. (Eds.), Development in Petrophysics. Geol. Soc. Spec. Publ., 122:299-344. doi:10.1144/GSL.SP.1997.122.01.18

Dick, H.J.B., Natland, J.H., Alt, J.C., Bach, W., Bideau, D., Gee, J.S., Haggas, S., Hertogen, J.G.H., Hirth, G., Holm, P.M., Ildefonse, B., Iturrino, G.J., John, B.E., Kelley, D.S., Kikawa, E., Kingdon, A., LeRoux, P.J., Maeda, J., Meyer, P.S., Miller, D.J., Naslund, H.R., Niu, Y.-L., Robinson, P.T., Snow, J., Stephen, R.A., Trimby, P.W., Worm, H.-U., and Yoshinobu, A., 2000. A long in situ section of the lower ocean crust: results of ODP Leg 176 drilling at the Southwest Indian Ridge. Earth Planet. Sci. Lett., 179(1):31-51. doi:10.1016/S0012-821X(00)00102-3

Dick, H.J.B., Natland, J.H., Miller, D.J., et al., 1999. Proc. ODP, Init. Repts., 176: College Station, TX (Ocean Drilling Program). doi:10.2973/odp.proc.ir.176.1999

Einaudi, F., Pezard, P.A., Cochemé, J.J., Coulon, C., Laverne, C., and Godard, M., 2000. Petrography, geochemistry and physical properties of a continuous extrusive section from the Sarami Massif, Semail ophiolite. Mar. Geoph. Res., 21(3/4):387-408. doi:10.1023/ A:1026752415989

Einaudi, F., Pezard, P.A., Ildefonse, B., and Glover, P., 2005. Electrical properties of slow-spreading gabbros from ODP Hole 1105A, SW Indian Ridge. In Harvey, P.K., and Brewer, T.S. (Eds.), Petrophysical Properties of Crystalline Rocks. Geol. Soc. Spec. Publ., 240(1):179-193. doi:10.1144/GSL.SP.2005.240.01.14

Gillis, K., Mével, C, Allan, J., et al., 1993. Proc. ODP, Init. Repts., 147: College Station, TX (Ocean Drilling Program). doi:10.2973/odp.proc.ir.147.1993

Goldberg, D., 1997. The role of downhole measurements in marine geology and geophysics. Rev. Geophys., 35(3):315-342. doi:10.1029/97RG00221

Guéguen, Y., and Palciauskas, V., 1992. Introduction à la physique des roches: Paris (Hermann).

Ildefonse, B., Blackman, D., John, B.E., Ohara, Y., Miller, D.J., MacLeod, C.J., and the IODP Expeditions 304-305 Scientists, 2006. IODP Expeditions $304 \& 305$ characterize the lithology, structure, and alteration of an oceanic core complex. Sci. Drill., 3:4-11. doi:10.2204/ iodp.sd.3.01.2006

Ildefonse, B., Blackman, D.K., John, B.E., Ohara, Y., Miller, D.J., MacLeod, C.J., and Integrated Ocean Drilling Program Expeditions 304/305 Science Party, 2007. Oceanic core complexes and crustal accretion at slow-spreading ridges. Geology, 35(7):623-626. doi:10.1130/G23531A.1

Ildefonse, B., and Pezard, P., 2001. Electrical properties of slow-spreading ridge gabbros from ODP Site 735, Southwest Indian Ridge. Tectonophysics, 330(1-2):69-92. doi:10.1016/S0040-1951(00)00220-1

Ildefonse, B., Valsardieu, B., Pezard, P., Mainprice, D., and Garrido, C.J., 1999. Petrophysics and anisotropy of gab- bros and peridotites from the Oman ophiolite. In Leiss, B., Ullemeyer, K., and Weber, K. (Eds.), Textures and Physical Properties of Rocks. Göettinger Arb. Geol. Paläontol., Geol. Inst., Univ. Göttingen, 73-74.

Kelemen, P.B., Kikawa, E., Miller, D.J., et al., 2004. Proc. ODP, Init. Repts., 209: College Station, TX (Ocean Drilling Program). doi.10.2973/odp.proc.ir.209.2004

McCaig, A.M., Cliff, R.A., Escartin, J., Fallick, A.E., and MacLeod, C.J., 2007. Oceanic detachment faults focus very large volumes of black smoker fluids. Geology, 35(10):935-938. doi:10.1130/G23657A.1

Pettigrew, T.L., Casey, J.F., Miller, D.J., et al., 1999. Proc. ODP, Init. Repts., 179: College Station, TX (Ocean Drilling Program). doi:10.2973/odp.proc.ir.179.1999

Pape, H., Riepe, L., and Schopper, J.R., 1985. Petrophysical detection of microfissures in granites. Trans. SPWLA 26th Annu. Logging Symp., Paper P.

Pezard, P.A., 1990. Electrical properties of mid-ocean ridge basalt and implications for the structure of the upper oceanic crust in Hole 504B. J. Geophys. Res., 95(B6):9237-9264. doi:10.1029/JB095iB06p09237

Pezard, P.A., Hermitte, D., Revil, A., Virlogeux, D., Leutsch, Y., and Harvey, P.K., 1999. Physical properties of granite, with application to nuclear waste storage in subsurface. In Leiss, B., Ullemeyer, K., and Weber, K. (Eds.), Textures and Physical Properties of Rocks. Göettinger Arb. Geol. Paläontol., Geol. Inst., Univ. Göttingen, 149-151.

Pezard, P.A., Howard, J.J., and Goldberg, D., 1991. Electrical conduction in oceanic gabbros, Hole 735B, Southwest Indian Ridge. In Von Herzen, R.P., Robinson, P.T., et al., Proc. ODP, Sci. Results, 118: College Station, TX (Ocean Drilling Program), 323-331. doi:10.2973/ odp.proc.sr.118.161.1991

Pezard, P.A., and Luthi, S.M., 1988. Borehole electrical images in the basement of the Cajon Pass scientific drillhole, California: fracture identification and tectonic implications. Geophys. Res. Lett., 15(9):1017-1020. doi:10.1029/GL015i009p01017

Revil, A. and Glover, P.W.J., 1998. Nature of surface electrical conductivity in natural sands, sandstones, and clays. Geoph. Res. Lett., 25(5):691-694. doi:10.1029/ 98GL00296

Revil, A., Hermitte, D., Spangenberg, E., and Cochemé, J.J., 2002. Electrica

Robinson, P.T, Von Herzen, R., et al., 1989. Proc. ODP, Init. Repts., 118: College Station, TX (Ocean Drilling Program). doi:10.2973/odp.proc.ir.118.1989

Walsh, J.B., and Brace, W.F., 1984. The effect of pressure on porosity and the transport properties of rocks. J. Geophys. Res., 89(B11):9425-9431. doi:10.1029/ JB089iB11p09425

Waxman, M.H., and Smits, L.J.M., 1968. Electrical conductivities in oil-bearing shaly sands. SPE J., 8(2):107-122. doi:10.2118/1863-A

Initial receipt: 27 June 2008

Acceptance: 9 July 2009

Publication: 8 September 2009

MS 304305-204 
Figure F1. Examples of plots of core conductivities as a function of saturating fluid conductivities (log measured conductivity $\left[C_{0}\right]$ and $\log$ water conductivity $\left.\left[C_{\mathrm{W}}\right]\right)$. Best-fit curves are calculated using Waxman and Smits (1968) (WS, orange curve) and Revil and Glover (1998) (RG, purple curve) models. A. Good fit example. B. Bad fit example (higher errors on $F$ and $C_{s}$ estimates, see Fig. F2). C. Good fit example with deflection of RG fit curve at low salinities, pointing to lower surface conductivities. D. Example of curve in which a good fit is obtained by removing a point (in this case, lowest salinity) that is several order of magnitudes out of range compared to adjacent point. See text for further explanations.

A
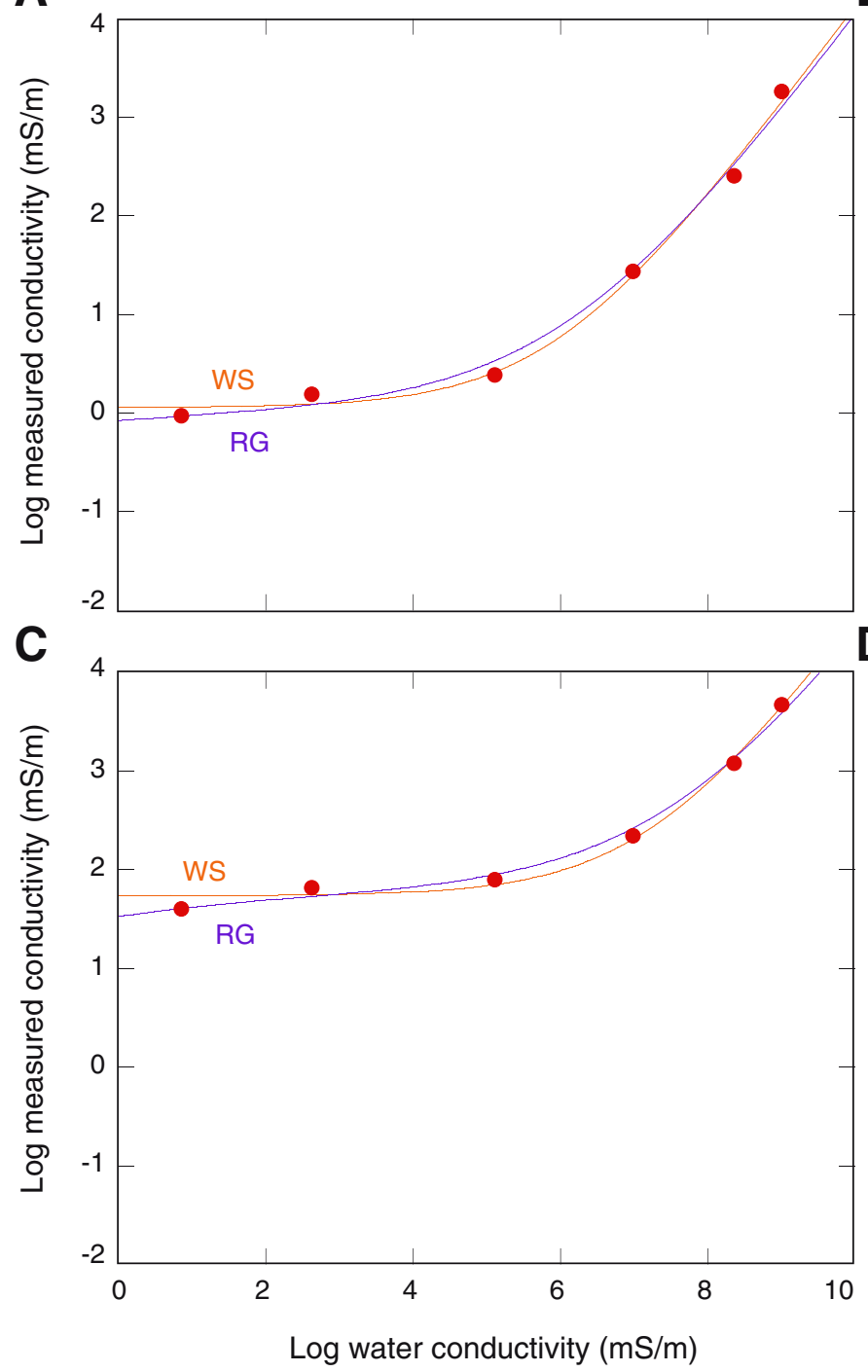

B

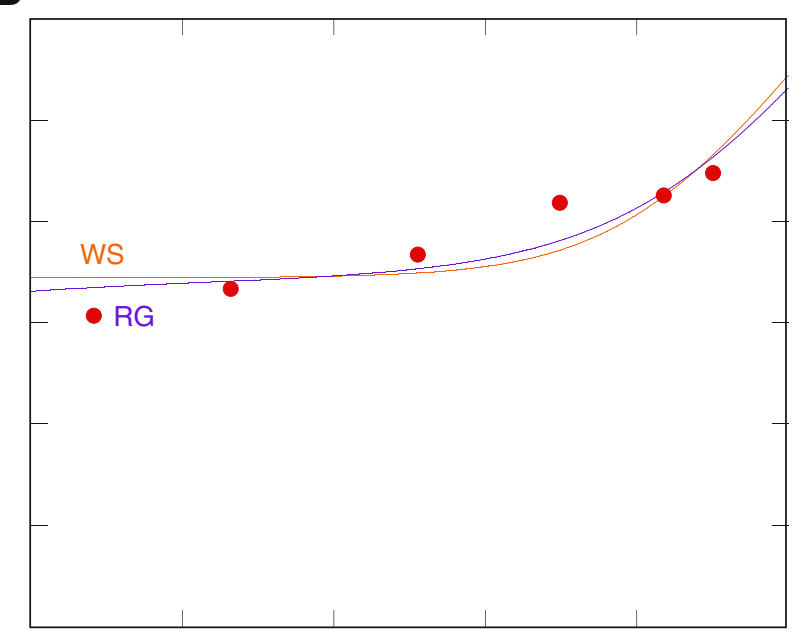

D

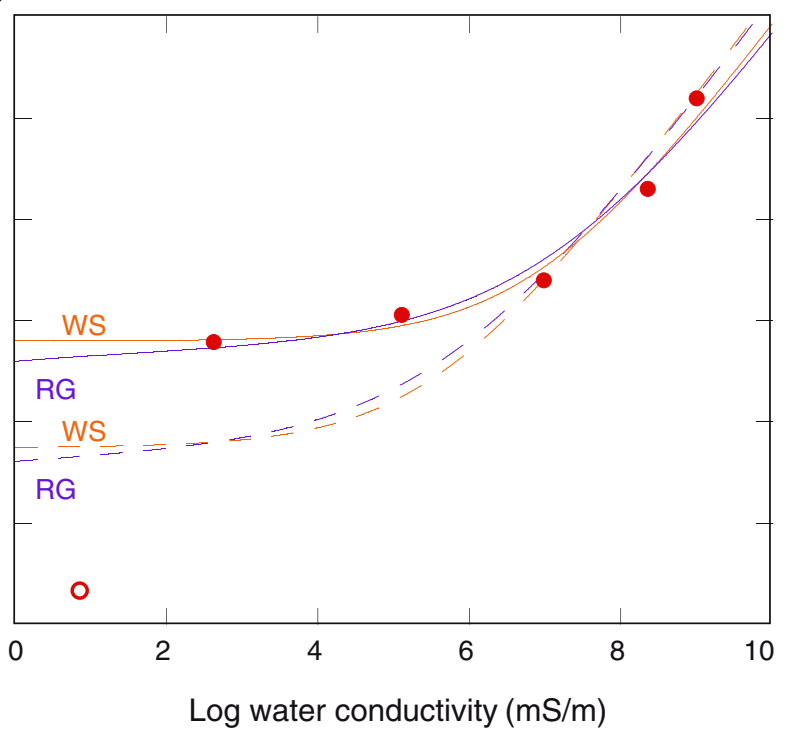


Figure F2. Downhole distributions of electrical formation factor $(F)$ and surface electrical conductivity $\left(C_{s}\right)$ calculated using Waxman and Smits (1968) and Revil and Glover (1998) models. Error bars show standard error obtained for $F$ and $C_{\mathrm{s}}$ from the fit curves (see examples in Fig. F1).

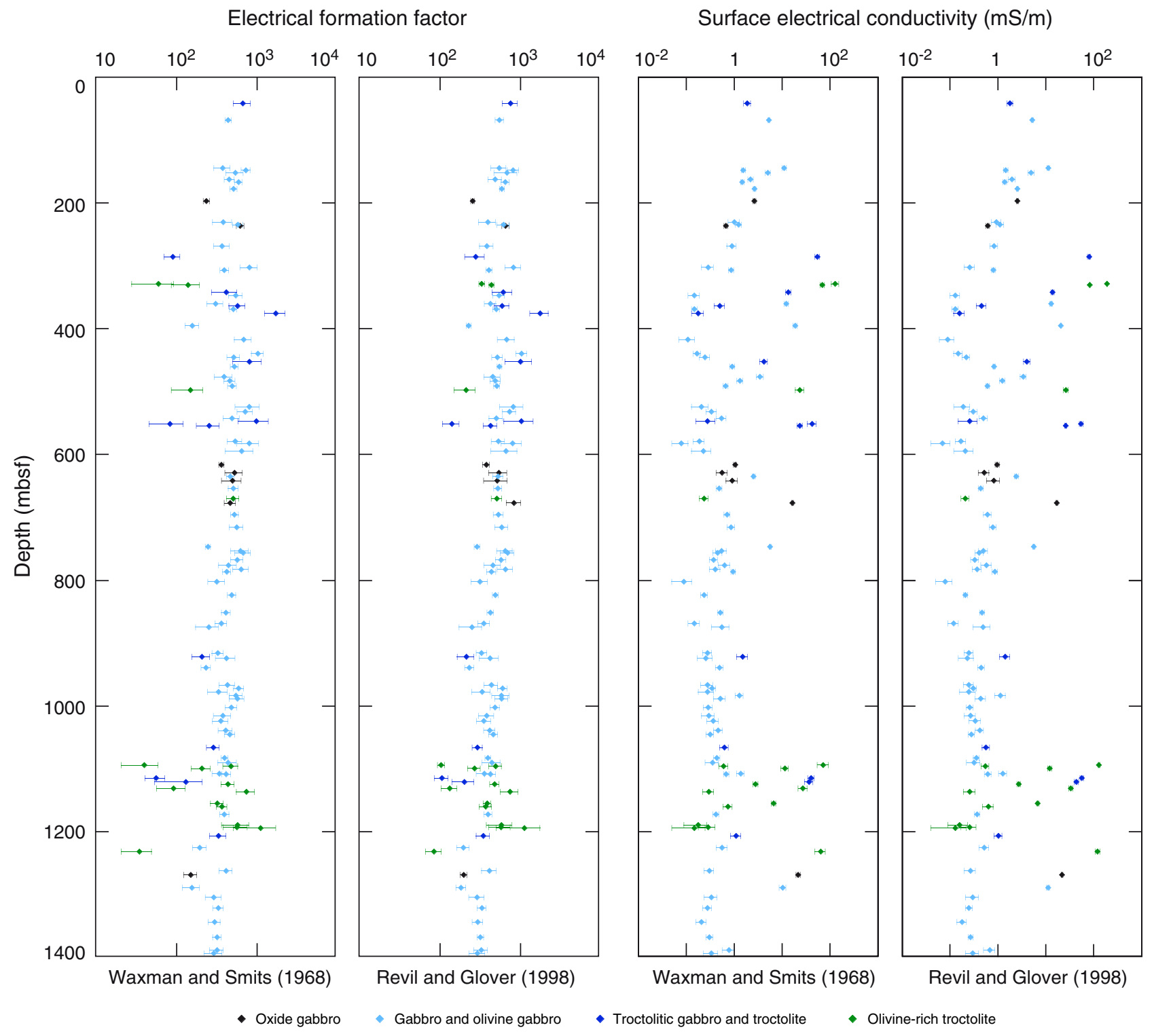


Figure F3. Compared downhole distributions of (A) lithology, (B) hand sample alteration, (C) borehole electrical resistivity, (D) porosity ( $\phi)$, (E) grain density, and (F-I) electrical properties of formation factor $(F)$, surface conductivity $\left(C_{S}\right)$, tortuosity $(\tau)$, and cementation index $(m)$. Open circles $=$ Waxman and Smits (1968) model, solid circles $=$ Revil and Glover (1998) model. LLS = shallow laterolog.

A $20 \mathrm{~m}$ running $\mathbf{B}$ Alteration (\%) average fraction shipboard Borehole electrical average fraction shipboard Borehole electrical

D $\quad$ E

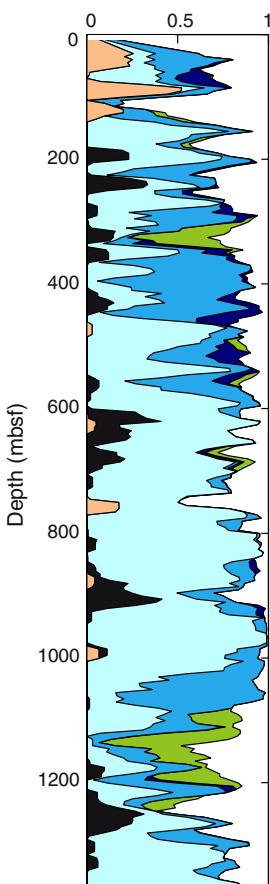

1400

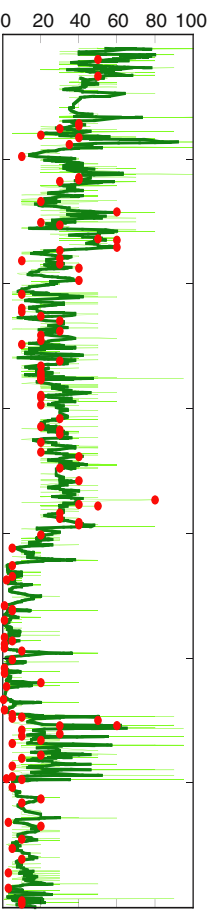

$\square$ Basaltoiabase
Oxide gabbro

All intervals
Sintervals running
average
sample internat Porosity

E

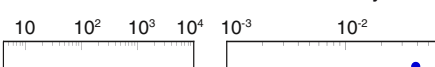

Electrical

G

Electrical surface $\mathbf{H}$

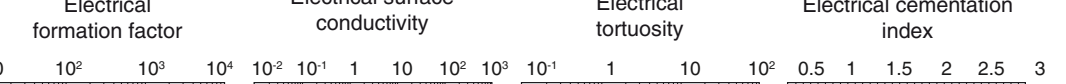

H Electrical

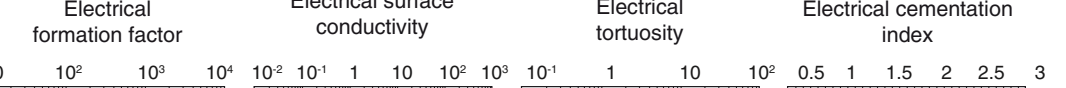

Electrical cementation

Grain density $\left(\mathrm{g} / \mathrm{cm}^{3}\right)$
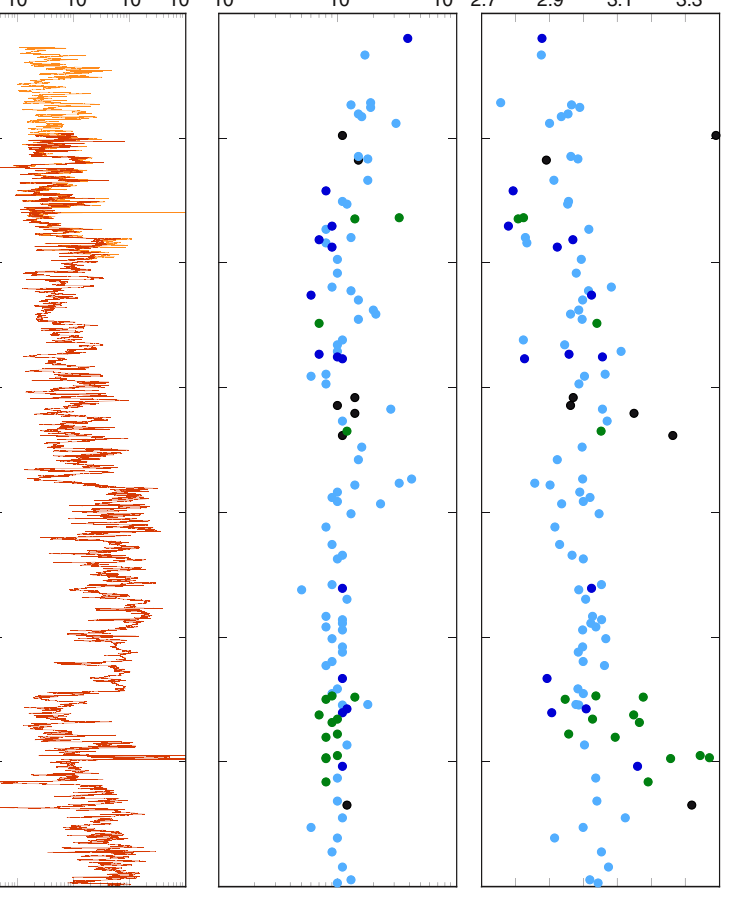

LLS, Pass 3

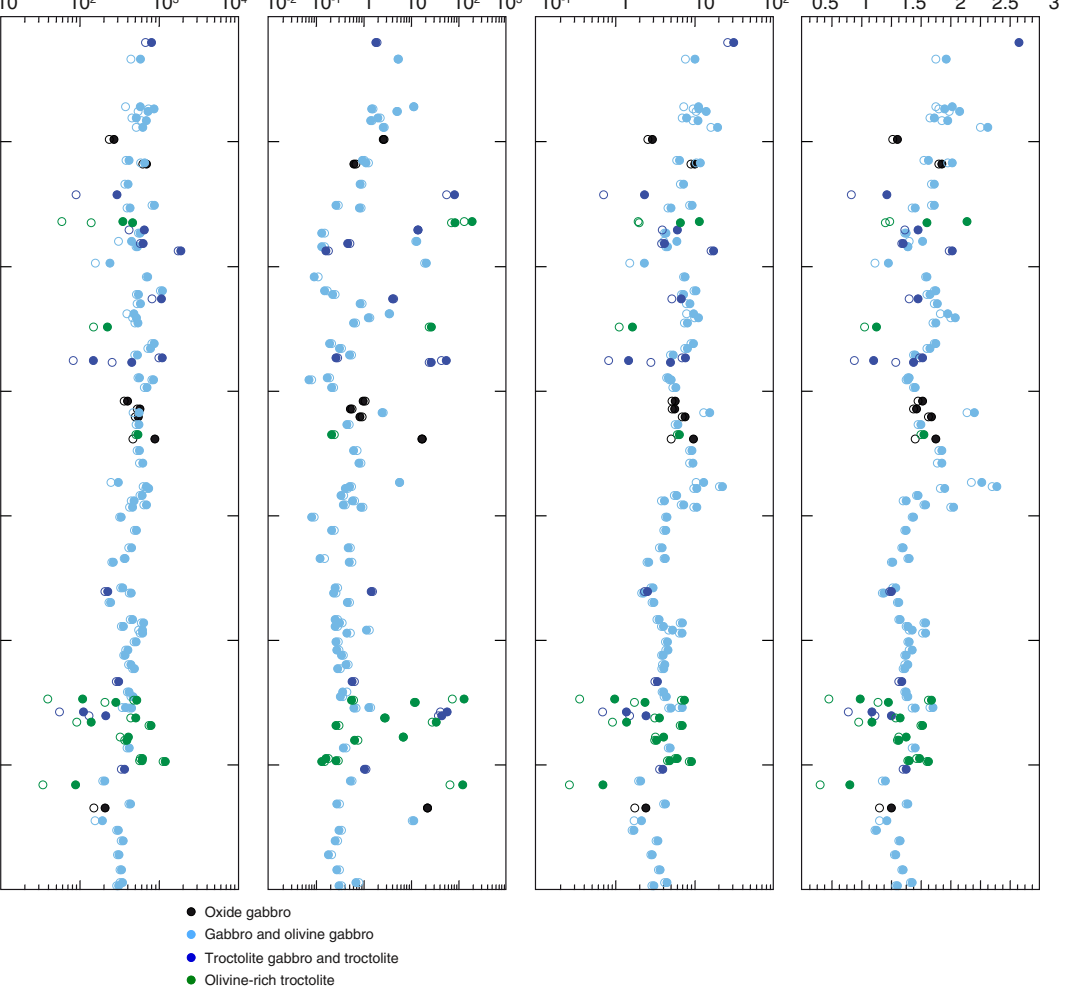

- Troctilitic gabbro
olvine gabbro

- Olivine-rich troctolite,
dunite, harbourgite

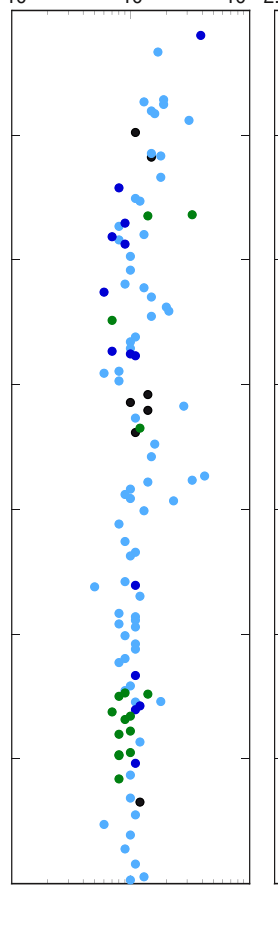

- Olivine-rich troctolili 
Figure F4. Variations of electrical properties with sample porosity $(\phi)$. A. Formation factor $(F)$. B. Surface conductivity $\left(C_{s}\right)$. C. Tortuosity $(\tau)$. D. Cementation index $(m)$. Electrical properties calculated from Revil and Glover (1998) model.

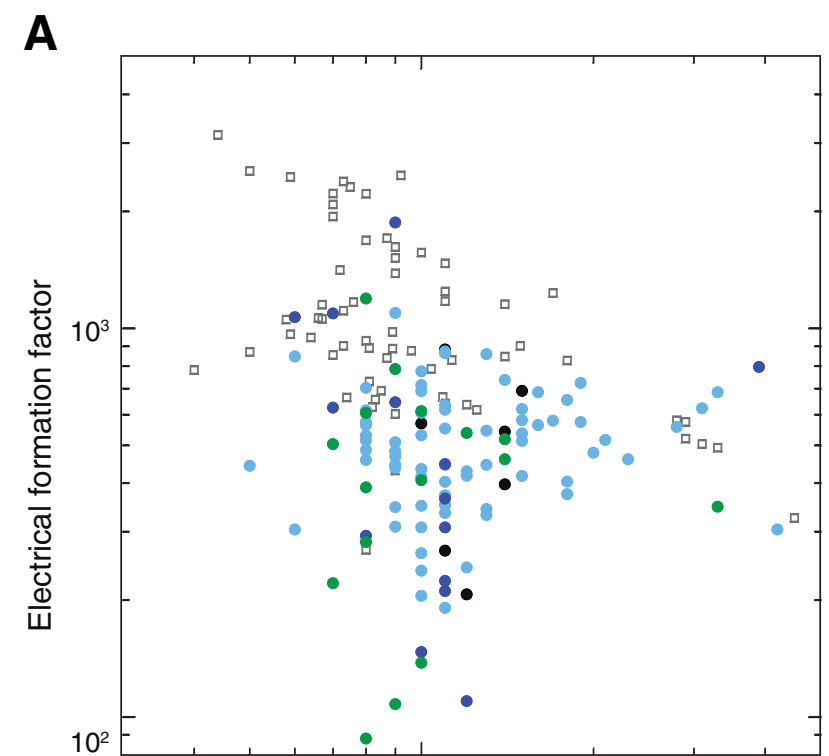

B

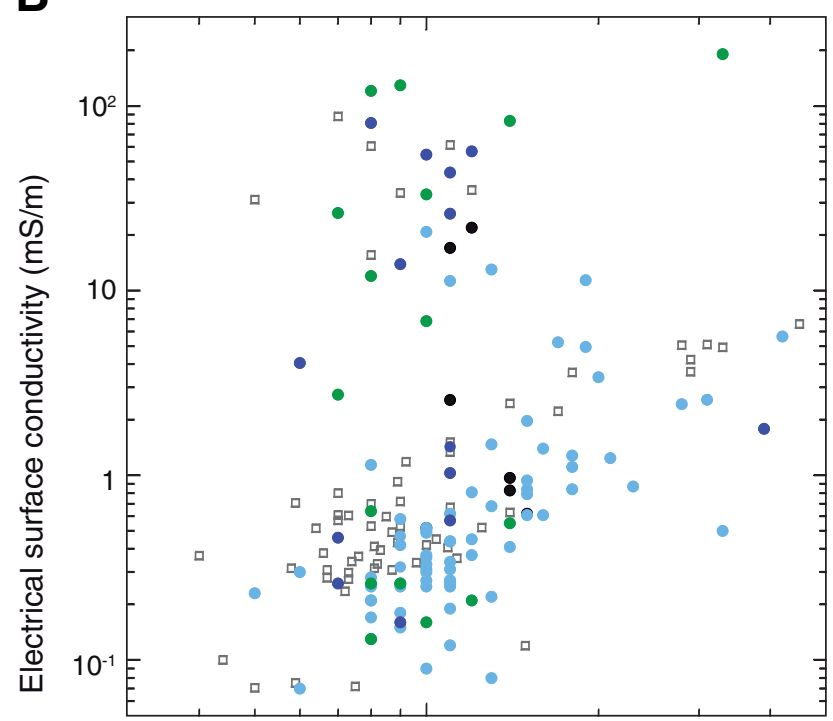

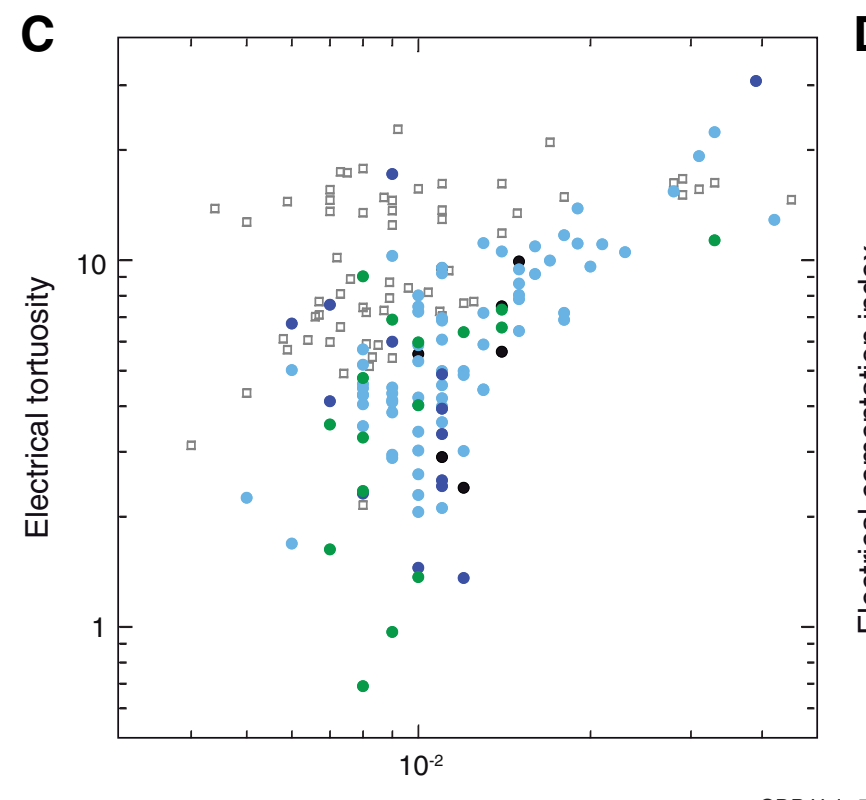

D
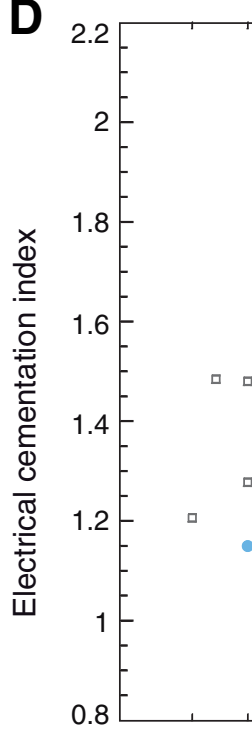

- ODP Hole 735B gabbroic rocks

- Oxide gabbro

- Gabbro and olivine gabbro

- Troctolitic gabbro and troctolite

- Olivine-rich troctolite

Porosity

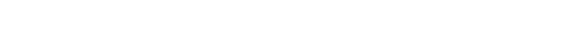


Figure F5. Variations of (A, B) porosity $(\phi),(\mathbf{C}, \mathbf{D})$ electrical tortuosity $(\tau)$, and $(\mathbf{E}, \mathbf{F})$ electrical cementation in$\operatorname{dex}(m)$ with cataclastic fabric intensity and degree of serpentinization. Cataclastic fabric intensity scale is the same as scale used during shipboard core descriptions (see the "Methods" chapter). Cataclastic fabric intensity and degree of serpentinization are those of intervals from which measured sample originated, as estimated onboard from macroscopic observation of hand samples. Electrical tortuosity $(\tau)$ and cementation index $(m)$ are calculated from Revil and Glover (1998) model.
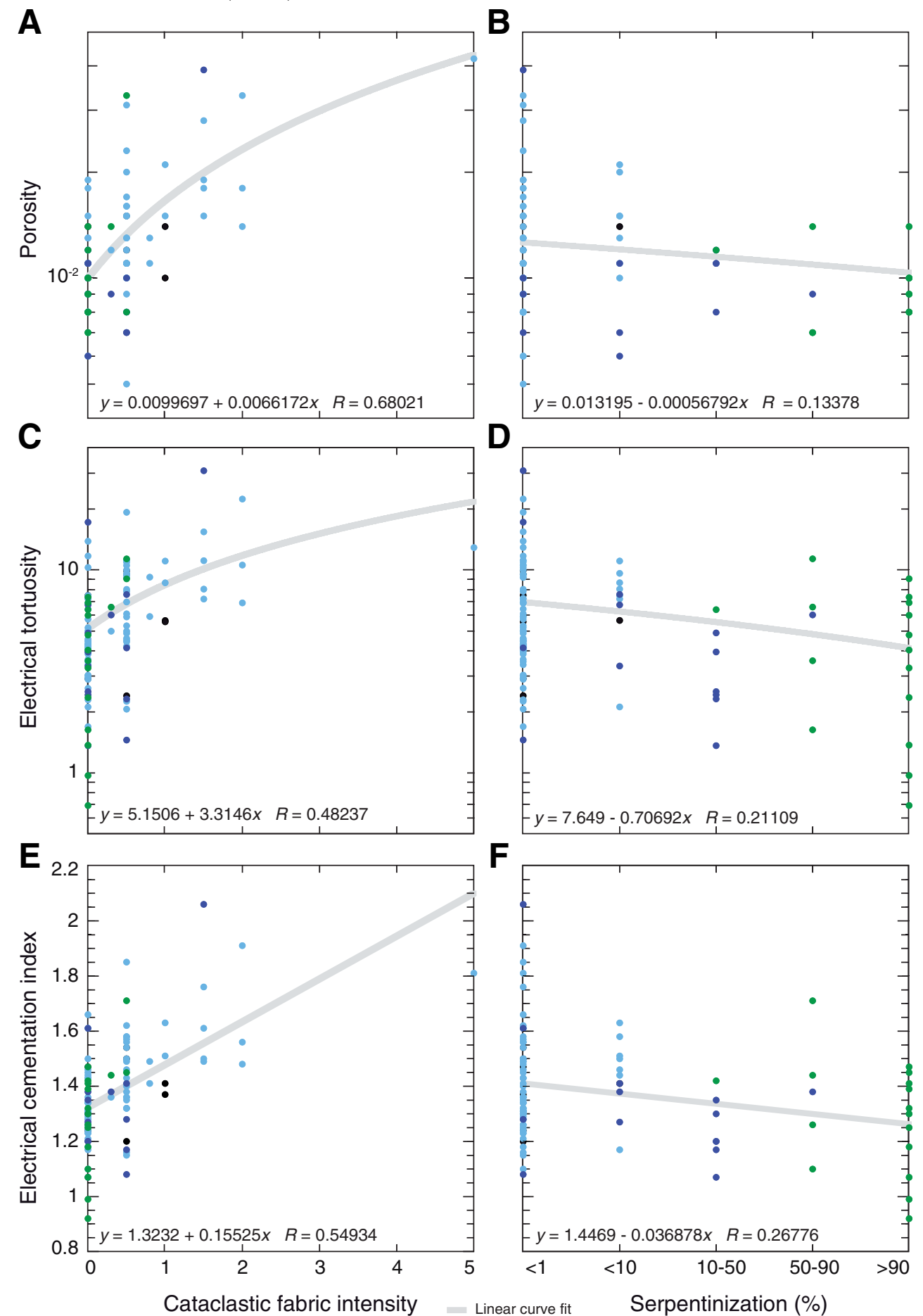

$\mathbf{F}$

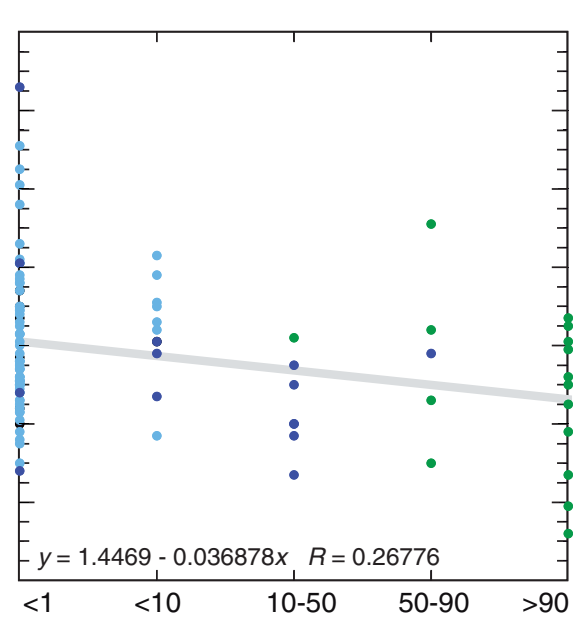

- Oxide gabbro

Serpentinization (\%)

- Gabbro and olivine gabbro

- Troctolitic gabbro and troctolite

- Olivine-rich troctolite 
Table T1. Sample resistivities at varying saturation fluid concentrations. (See table notes.) (Continued on next page.)

\begin{tabular}{|c|c|c|c|c|c|c|c|c|c|}
\hline \multirow{2}{*}{$\begin{array}{l}\text { Core, section, } \\
\text { interval (cm) }\end{array}$} & \multirow{2}{*}{$\begin{array}{l}\text { Depth } \\
\text { (mbsf) }\end{array}$} & \multirow[b]{2}{*}{ Lithology } & \multirow{2}{*}{$\begin{array}{l}\text { Batch } \\
\text { number }\end{array}$} & \multicolumn{6}{|c|}{ Resistivity $(\Omega \mathrm{m})$} \\
\hline & & & & $\sim 0.006 \mathrm{~g} / \mathrm{L}$ & $\sim 0.06 \mathrm{~g} / \mathrm{L}$ & $\sim 0.6 \mathrm{~g} / \mathrm{L}$ & $\sim 6 \mathrm{~g} / \mathrm{L}$ & $\sim 30 \mathrm{~g} / \mathrm{L}$ & $\sim 60 \mathrm{~g} / \mathrm{L}$ \\
\hline \multicolumn{10}{|l|}{ 304-U1309D- } \\
\hline $5 R-3,124-126$ & 40.11 & Troctolitic gabbro & 5 & 725.1 & 494.0 & 416.6 & 188.9 & 143.8 & 77.4 \\
\hline $11 \mathrm{R}-2,48-50$ & 66.77 & Gabbro & 1 & 207.7 & 175.1 & 162.2 & 127.8 & 71.2 & 39.7 \\
\hline $25 \mathrm{R}-1,33-35$ & 143.03 & Gabbro & 1 & 103.5 & 87.9 & 84.4 & 64.0 & 35.4 & 37.3 \\
\hline $25 R-3,94-96$ & 146.49 & Olivine-bearing gabbro & 3 & 714.5 & 593.5 & 464.2 & 402.2 & 133.1 & 73.3 \\
\hline $26 \mathrm{R}-3,83-85$ & 150.94 & Olivine-bearing gabbro & 3 & 216.1 & 185.3 & 173.4 & 129.5 & 110.0 & 38.6 \\
\hline $28 R-4,45-47$ & 161.38 & Gabbro & 1 & 5277.5 & 453.8 & 346.9 & 246.9 & 100.0 & 40.9 \\
\hline $29 R-3,64-66$ & 165.45 & Gabbro & 1 & 731.3 & 608.1 & 570.0 & 269.7 & 137.1 & 59.9 \\
\hline 32R-1, 9-11 & 176.39 & Olivine-bearing gabbro & 3 & 436.9 & 350.1 & 305.4 & 192.5 & 86.5 & 59.4 \\
\hline $36 \mathrm{R}-1,10-12$ & 195.7 & Oxide gabbro & 1 & 443.9 & 334.2 & 276.0 & 126.3 & 46.5 & 29.9 \\
\hline $43 \mathrm{R}-1,57-79$ & 229.37 & Olivine gabbro & 2 & 1428.5 & 1021.2 & 353.4 & 247.9 & 104.6 & 45.3 \\
\hline $44 \mathrm{R}-1,18-20$ & 233.38 & Olivine gabbro & 2 & 37778.9 & 728.0 & 697.7 & 342.2 & 91.6 & 78.2 \\
\hline $44 \mathrm{R}-2,83-85$ & 235.53 & Disseminated oxide gabbro & 1 & 1728.6 & 1277.8 & 966.4 & 433.6 & - & 72.5 \\
\hline $51 \mathrm{R}-1,74-76$ & 267.54 & Gabbro & 1 & 1568.9 & 964.5 & 532.4 & 206.2 & 72.8 & 64.8 \\
\hline $54 R-3,45-47$ & 284.34 & Troctolite & 4 & 19.7 & 17.4 & 267.6 & 959.4 & 8.2 & 7.7 \\
\hline $58 \mathrm{R}-1,110-112$ & 301.5 & Olivine gabbro & 2 & 5241.2 & 2615.9 & 1473.3 & 444.1 & 269.2 & 99.8 \\
\hline $59 \mathrm{R}-1,58-60$ & 305.78 & Olivine gabbro & 2 & 1403.7 & 1047.0 & 579.3 & 281.4 & 85.7 & 50.1 \\
\hline $63 R-3,41-43$ & 327.77 & Olivine-rich troctolite & 5 & 11.3 & 7.4 & 5.5 & 256.6 & 4.3 & 4.2 \\
\hline $64 \mathrm{R}-1,58-60$ & 329.78 & Olivine-rich troctolite & 5 & 19.2 & 13.8 & 12.3 & 10.8 & 9.5 & 8.3 \\
\hline $66 \mathrm{R}-2,93-95$ & 341.23 & Troctolite & 4 & 98.9 & 76.9 & 59.5 & 48.3 & 37.7 & 34.7 \\
\hline 67R-3, 17-19 & 346.43 & Gabbro (fine-grained) & 1 & 9532.0 & 4014.0 & 1493.2 & 535.6 & 135.7 & 74.1 \\
\hline 70R-2, 76-78 & 359.45 & Olivine gabbro & 2 & 103.8 & 73.1 & 65.5 & 244.8 & 38.0 & 26.0 \\
\hline $71 \mathrm{R}-1,14-16$ & 362.94 & Troctolitic gabbro & 4 & 2626.2 & 1664.2 & 1026.1 & 299.9 & 202.0 & 61.9 \\
\hline $71 \mathrm{R}-5,13-15$ & 368.26 & Olivine gabbro & 2 & 6903.0 & 5466.3 & 1798.4 & 592.0 & 112.6 & 55.1 \\
\hline $73 \mathrm{R}-2,86-88$ & 374.71 & Troctolitic gabbro & 4 & 8066.5 & 5897.7 & 1696.8 & 1397.9 & 386.5 & 242.7 \\
\hline $77 R-3,21-23$ & 394.34 & Olivine gabbro & 2 & 65.2 & 55.0 & 43.4 & 30.7 & 22.2 & 15.1 \\
\hline \multicolumn{10}{|l|}{ 305-U1309D- } \\
\hline $83 \mathrm{R}-2,29-31$ & 416.44 & Olivine gabbro & 2 & 10810.9 & 6873.9 & 2266.8 & 808.7 & 89.8 & 132.4 \\
\hline $88 \mathrm{R}-1,2-4$ & 439.02 & Olivine gabbro & 2 & 8861.1 & 4127.7 & 2438.6 & 800.9 & 271.5 & 126.6 \\
\hline 89R-1, 92-94 & 444.72 & Olivine gabbro & 2 & 5435.1 & 3058.1 & 1158.8 & 453.7 & 108.6 & 81.5 \\
\hline $90 \mathrm{R}-3,8-10$ & 451.68 & Troctolite & 4 & 342.8 & 263.0 & 186.9 & 112.4 & 104.5 & 83.8 \\
\hline $92 \mathrm{R}-1,123-125$ & 459.43 & Olivine-bearing gabbro & 3 & 1350.7 & 998.8 & 657.9 & 314.2 & 118.2 & 63.6 \\
\hline $95 R-3,35-37$ & 475.73 & Olivine-bearing gabbro & 3 & 352.5 & 279.7 & 251.3 & 110.9 & 57.3 & 58.5 \\
\hline $97 R-1,6-8$ & 482.26 & Olivine-bearing gabbro & 3 & 1063.2 & 583.1 & 504.8 & 261.7 & 100.5 & 53.4 \\
\hline 98R-3, 96-98 & 490.66 & Olivine-bearing gabbro & 3 & 1888.3 & 1310.9 & 837.7 & 297.3 & 123.0 & 62.2 \\
\hline 100R-1, 32-34 & 496.92 & Olivine-rich troctolite & 5 & 69.0 & 43.4 & 31.1 & 21.3 & 18.4 & 15.3 \\
\hline $105 \mathrm{R}-3,46-48$ & 523.65 & Olivine gabbro & 2 & 6889.7 & 3916.6 & 1497.9 & 431.8 & 408.8 & 80.2 \\
\hline $107 \mathrm{R}-2,52-54$ & 531.72 & Olivine-bearing gabbro & 3 & 3731.1 & 2812.9 & 1116.5 & 478.4 & 241.4 & 77.1 \\
\hline 109R-2, 69-71 & 541.84 & Olivine-bearing gabbro & 3 & 2563.7 & 1692.9 & 672.6 & 320.8 & 149.7 & 58.2 \\
\hline $110 \mathrm{R}-2,74-76$ & 546.76 & Troctolite & 4 & 5304.5 & 3388.8 & 1530.6 & 284.4 & 373.7 & 175.1 \\
\hline $111 \mathrm{R}-2,17-19$ & 550.87 & Troctolitic gabbro & 4 & 37.6 & 26.7 & 15.9 & 11.7 & 10.0 & 8.6 \\
\hline $111 \mathrm{R}-4,19-21$ & 553.76 & Troctolite & 4 & 54.8 & 40.9 & 32.4 & 94.0 & 23.6 & 18.8 \\
\hline $117 \mathrm{R}-1,55-57$ & 578.75 & Olivine gabbro & 2 & 6905.9 & 3676.2 & 1455.3 & 556.2 & 89.9 & 91.8 \\
\hline 117R-4, 27-29 & 582.03 & Gabbro (fine-grained) & 1 & 22045.5 & 6385.3 & 1930.6 & 600.3 & 277.0 & 128.9 \\
\hline $120 \mathrm{R}-2,26-28$ & 594.04 & Olivine-bearing gabbro & 3 & 6834.3 & 3534.0 & 1159.3 & 252.8 & 230.7 & 133.6 \\
\hline 124R-4, 47-49 & 615.87 & Oxide gabbro & 1 & 1025.5 & 824.4 & 677.5 & 236.6 & 89.8 & 38.2 \\
\hline 127R-2, 99-101 & 628.69 & Oxide gabbro & 1 & 2724.1 & 1042.8 & 1098.8 & 426.3 & 85.3 & 79.7 \\
\hline $128 \mathrm{R}-3,48-50$ & 634.39 & Gabbro & 1 & 441.2 & 356.5 & 299.0 & 240.6 & 83.2 & 48.4 \\
\hline 130R-1, 44-46 & 641.04 & Oxide gabbro & 1 & 1148.3 & 941.3 & 679.8 & 658.7 & 86.1 & 44.2 \\
\hline $132 \mathrm{R}-3,106-108$ & 653.72 & Gabbro & 1 & 2495.5 & 1762.1 & 1104.4 & 295.1 & 144.5 & 63.5 \\
\hline 136R-1, 32-34 & 669.72 & Olivine-rich troctolite & 5 & 5047.9 & 3522.8 & 1200.1 & 371.3 & 169.3 & 59.8 \\
\hline $137 R-2,126-128$ & 676.86 & Oxide gabbro & 1 & 65.6 & 60.3 & 57.2 & 47.3 & 42.8 & 28.2 \\
\hline 141R-2, 63-65 & 695.45 & Gabbro & 4 & 8746.2 & 1325.8 & 959.2 & 386.9 & 135.2 & 49.8 \\
\hline $145 \mathrm{R}-3,38-40$ & 715.67 & Olivine gabbro & 2 & 1423.2 & 1058.5 & 712.9 & 311.9 & 176.5 & 52.2 \\
\hline $152 \mathrm{R}-1,35-37$ & 746.55 & Gabbro (cataclastic) & 1 & 199.9 & 161.3 & 148.8 & 95.4 & 45.7 & 25.2 \\
\hline $153 \mathrm{R}-2,88-90$ & 753.38 & Gabbro & 1 & 2562.9 & 1654.7 & 865.8 & 294.0 & 201.8 & 78.4 \\
\hline $154 \mathrm{R}-1,30-32$ & 756.1 & Gabbro (cataclastic) & 1 & 2773.4 & 2073.4 & 1076.3 & 341.6 & 228.5 & 77.9 \\
\hline 157R-2, 75-77 & 767.52 & Olivine-bearing gabbro & 3 & 3467.7 & 2314.1 & 986.3 & 546.9 & 125.3 & 69.7 \\
\hline 159R-1, 104-106 & 776.04 & Gabbro & 1 & 2468.4 & 1000.7 & 981.3 & 220.4 & 105.4 & 69.3 \\
\hline $160 \mathrm{R}-2,117-119$ & 782.47 & Gabbro & 1 & 2967.8 & 2265.3 & 1083.9 & 568.5 & 93.8 & 112.6 \\
\hline $161 \mathrm{R}-2,44-46$ & 786.43 & Olivine gabbro & 2 & 1239.5 & 853.1 & 693.0 & 321.2 & 99.6 & 42.2 \\
\hline 164R-3, 110-112 & 802.29 & Gabbro & 1 & 11910.6 & 6366.0 & 941.9 & 206.0 & 115.9 & 56.7 \\
\hline $169 \mathrm{R}-1,64-66$ & 823.64 & Gabbro & 1 & 4951.5 & 3445.8 & 1272.2 & 388.9 & 125.4 & 65.8 \\
\hline $174 \mathrm{R}-4,53-55$ & 851.51 & Olivine-bearing gabbro & 3 & 2086.0 & 1945.2 & 812.6 & 287.6 & 90.5 & 62.4 \\
\hline $178 \mathrm{R}-3,8-10$ & 868.87 & Gabbro & 1 & 7154.6 & 4867.2 & 1526.7 & 520.5 & 62.4 & 39.9 \\
\hline 170R-4, 77-79 & 874.89 & Olivine gabbro & 2 & 3702.4 & 746.5 & 814.5 & 191.8 & 63.0 & 31.7 \\
\hline 188R-2, 37-39 & 915.81 & Olivine-bearing gabbro & 3 & 3618.1 & 3386.7 & 1009.5 & 196.6 & 86.7 & 55.6 \\
\hline
\end{tabular}


Table T1 (continued).

\begin{tabular}{|c|c|c|c|c|c|c|c|c|c|}
\hline \multirow{2}{*}{$\begin{array}{l}\text { Core, section, } \\
\text { interval }(\mathrm{cm})\end{array}$} & \multirow{2}{*}{$\begin{array}{l}\text { Depth } \\
\text { (mbsf) }\end{array}$} & \multirow[b]{2}{*}{ Lithology } & \multirow{2}{*}{$\begin{array}{c}\text { Batch } \\
\text { number }\end{array}$} & \multicolumn{6}{|c|}{ Resistivity $(\Omega \mathrm{m})$} \\
\hline & & & & $\sim 0.006 \mathrm{~g} / \mathrm{L}$ & $\sim 0.06 \mathrm{~g} / \mathrm{L}$ & $\sim 0.6 \mathrm{~g} / \mathrm{L}$ & $\sim 6 \mathrm{~g} / \mathrm{L}$ & $\sim 30 \mathrm{~g} / \mathrm{L}$ & $\sim 60 \mathrm{~g} / \mathrm{L}$ \\
\hline $189 \mathrm{R}-3,40-42$ & 922.09 & Troctolitic gabbro & 4 & 1134.9 & 403.5 & 358.8 & 132.0 & 57.6 & 22.9 \\
\hline 190R-1, 31-33 & 924.11 & Gabbro & 1 & 4660.8 & 3385.3 & 963.1 & 337.7 & 198.3 & 34.4 \\
\hline 194R-2, 27-29 & 939.15 & Gabbro & 1 & 2559.7 & 1332.5 & 773.2 & 224.4 & 58.2 & 24.0 \\
\hline 200R-2, 31-33 & 966.67 & Gabbro & 1 & 4745.8 & 2946.7 & 935.2 & 293.4 & 154.1 & 54.0 \\
\hline 202R-1, 40-42 & 972.4 & Gabbro & 1 & 3415.8 & 2593.5 & 1105.8 & 586.7 & 122.5 & 74.5 \\
\hline 203R-1, 99-101 & 977.79 & Gabbro & 1 & 5096.1 & 2642.4 & 683.5 & 303.7 & 135.9 & 33.1 \\
\hline 204R-2, 97-99 & 983.75 & Olivine-bearing gabbro & 3 & 4749.8 & 633.7 & 768.9 & 303.5 & 139.7 & 48.8 \\
\hline $205 R-2,83-85$ & 988.55 & Olivine gabbro & 2 & 76002.3 & 2228.5 & 1043.5 & 281.1 & 147.5 & 85.2 \\
\hline 208R-2, 74-76 & 1002.84 & Olivine-bearing gabbro & 3 & 4814.6 & 2439.9 & 1163.6 & 401.1 & 116.1 & 65.1 \\
\hline $211 \mathrm{R}-1,64-66$ & 1015.84 & Olivine-bearing gabbro & 3 & 4430.3 & 2700.9 & 872.6 & 208.6 & 122.6 & 62.4 \\
\hline $212 R-4,65-67$ & 1024.19 & Olivine gabbro & 2 & 3278.5 & 2035.8 & 26914.0 & 187.3 & 107.6 & 49.9 \\
\hline 216R-1, 17-19 & 1039.37 & Olivine gabbro & 2 & 3058.6 & 1724.9 & 751.4 & 297.7 & 119.3 & 50.4 \\
\hline $217 \mathrm{R}-2,23-25$ & 1045.64 & Olivine gabbro & 2 & 3762.8 & 2690.5 & 1044.2 & 459.5 & 90.2 & 64.5 \\
\hline $221 \mathrm{R}-3,64-66$ & 1066.42 & Troctolitic gabbro & 4 & 2174.9 & 1298.9 & 585.1 & 184.6 & 72.6 & 43.9 \\
\hline $225 \mathrm{R}-1,72-74$ & 1083.12 & Olivine gabbro & 2 & 81384.6 & 2329.5 & 981.2 & 340.9 & 80.0 & 54.3 \\
\hline $226 \mathrm{R}-3,40-42$ & 1090.5 & Gabbro & 1 & 3745.5 & 1999.9 & 1306.2 & 263.9 & 72.1 & 99.4 \\
\hline $227 \mathrm{R}-2,82-84$ & 1094.25 & Olivine-rich troctolite & 5 & 22.8 & 13.3 & 7.8 & 279.6 & 4.8 & 4.2 \\
\hline $227 R-3,124-126$ & 1096.17 & Olivine-rich troctolite & 5 & 2349.1 & 1391.5 & 779.2 & 258.1 & 107.2 & 79.5 \\
\hline 228R,3, 29-31 & 1099.8 & Olivine-rich troctolite & 5 & 111.5 & 184.3 & 73.1 & 46.4 & 27.1 & 24.3 \\
\hline $230 \mathrm{R}, 2,125-127$ & 1108.2 & Olivine-bearing gabbro & 3 & 800.7 & 685.8 & 441.4 & 245.2 & 102.7 & 27.5 \\
\hline $230 \mathrm{R}-3,28-30$ & 1108.66 & Gabbro & 1 & 1489.8 & 1286.2 & 903.8 & 421.7 & 75.2 & 46.0 \\
\hline 232R-1, 15-17 & 1115.25 & Troctolitic gabbro & 4 & 34.9 & 21.4 & 21.6 & 12.3 & 7.6 & 6.6 \\
\hline 233R-1, 141-143 & 1121.31 & Troctolitic gabbro & 4 & 40.9 & 29.9 & 19.7 & 15.3 & 90.4 & 11.3 \\
\hline 234R-1, 27-29 & 1124.97 & Olivine-rich troctolite & 5 & 461.3 & 318.4 & 255.9 & 407.3 & 75.3 & 50.3 \\
\hline 235R-2, 91-93 & 1131.67 & Olivine-rich troctolite & 5 & 62.5 & 34.2 & 23.1 & 21.4 & 11.9 & 10.5 \\
\hline $236 \mathrm{R}-2,125-127$ & 1136.9 & Olivine-rich troctolite & 5 & 4525.7 & 2789.6 & 1170.9 & 925.8 & 180.6 & 68.0 \\
\hline 240R-2, 72-74 & 1155.46 & Olivine-rich troctolite & 5 & 185.5 & 142.3 & 112.1 & 452.2 & 49.3 & 32.2 \\
\hline 241R-2, 89-91 & 1160.66 & Olivine-rich troctolite & 5 & 3929.0 & 1164.6 & 944.0 & 282.3 & 59.9 & 53.9 \\
\hline $244 \mathrm{R}-1,34-36$ & 1173.04 & Gabbro & 1 & 2871.3 & 2064.0 & 867.8 & 391.7 & 85.1 & 48.8 \\
\hline $247 R-3,16-18$ & 1190.05 & Olivine-rich troctolite & 5 & 11963.7 & 2781.0 & 1004.7 & 453.4 & 171.8 & 97.7 \\
\hline $248 \mathrm{R}-2,22-24$ & 1193.36 & Olivine-rich troctolite & 5 & 7014.6 & 2016.7 & 1000.5 & 366.8 & 163.7 & 89.5 \\
\hline $248 R-3,36-38$ & 1194.85 & Olivine-rich troctolite & 5 & 15361.2 & 2511.1 & 4336.2 & 299.2 & 474.0 & 179.7 \\
\hline $251 \mathrm{R}-1,87-89$ & 1207.17 & Troctolitic gabbro & 4 & 1346.0 & 819.5 & 393.3 & 182.1 & 82.5 & 47.5 \\
\hline $255 \mathrm{R}-1,53-55$ & 1226.03 & Olivine gabbro & 2 & 2359.7 & 1217.3 & 560.1 & 139.4 & 69.0 & 20.7 \\
\hline $256 \mathrm{R}-2,83-85$ & 1232.27 & Olivine-rich troctolite & 5 & 23.1 & 16.7 & 8.6 & 344.2 & 4.5 & 3.9 \\
\hline $262 \mathrm{R}-3,112-114$ & 1263.07 & Gabbro & 1 & 2755.6 & 3527.7 & 1268.9 & 559.3 & 73.2 & 43.5 \\
\hline $264 R-1,81-83$ & 1269.51 & Oxide gabbro & 1 & 7865.9 & 54.2 & 40.2 & 28.7 & 19.9 & 13.9 \\
\hline $268 \mathrm{R}-2,83-85$ & 1290.06 & Olivine gabbro & 2 & 130.3 & 92.9 & 62.9 & 274.9 & 25.7 & 16.7 \\
\hline 271R-3, 59-61 & 1305.25 & Gabbro & 1 & 3926.3 & 1974.7 & 865.0 & 374.9 & 44.5 & 43.0 \\
\hline $275 R-3,88-90$ & 1322.38 & Gabbro & 1 & 4520.4 & 2684.3 & 953.0 & 235.3 & 97.5 & 46.4 \\
\hline 280R-1, 88-90 & 1344.32 & Olivine-bearing gabbro & 3 & 6665.2 & 2716.6 & 960.7 & 278.2 & 73.9 & 41.5 \\
\hline 285R-1, 90-92 & 1368.9 & Gabbro & 1 & 4114.3 & 2337.7 & 996.1 & 273.5 & 88.1 & 37.2 \\
\hline 290R-2, 89-91 & 1389.23 & Olivine-bearing gabbro & 3 & 5420.5 & 1254.9 & 799.0 & 153.2 & 85.0 & 45.4 \\
\hline 291R-3, 39-41 & 1394.39 & Olivine gabbro & 2 & 4300.2 & 2046.5 & 718.5 & 183.1 & 109.0 & 38.6 \\
\hline
\end{tabular}

Notes: See Table $\mathbf{T} 2$ for batch number data. $-=$ no data. 
Table T2. Saturating fluid resistivities. (See table note.)

\begin{tabular}{ccccccc}
\hline & \multicolumn{6}{c}{ Resistivity $(\Omega \mathrm{m})$} \\
\cline { 2 - 7 } Batch number & $\sim 0.006 \mathrm{~g} / \mathrm{L}$ & $\sim 0.06 \mathrm{~g} / \mathrm{L}$ & $\sim 0.6 \mathrm{~g} / \mathrm{L}$ & $\sim 6 \mathrm{~g} / \mathrm{L}$ & $\sim 30 \mathrm{~g} / \mathrm{L}$ & $\sim 60 \mathrm{~g} / \mathrm{L}$ \\
\hline 1 & 427.4 & 72.46 & 6.068 & 0.9272 & 0.2347 & 0.1233 \\
2 & 440.5 & 73.53 & 6.057 & 0.9275 & 0.2353 & 0.1230 \\
3 & 432.9 & 72.99 & 6.079 & 0.9273 & 0.2358 & 0.1227 \\
4 & 436.7 & 71.94 & 6.079 & 0.9272 & 0.2347 & 0.1227 \\
5 & 438.6 & 73.53 & 6.075 & 0.9301 & 0.2353 & 0.1230 \\
\hline
\end{tabular}

Note: Deviations from normal resistivity at the given fluid salinity are due to progressive reequilibration between the sample and fluid before measurements are taken. 
Table T3. Physical and electrical properties. This table is available in an oversized format. 
Plate P1. Photomicrographs of sample thin sections in plane- (PPL) and cross-polarized light (CPL). Thin sections were obtained after chips were taken from the minicore ends; each section is $\sim 2.54 \mathrm{~cm}$ in diameter (1 inch). (Continued on next ten pages.)

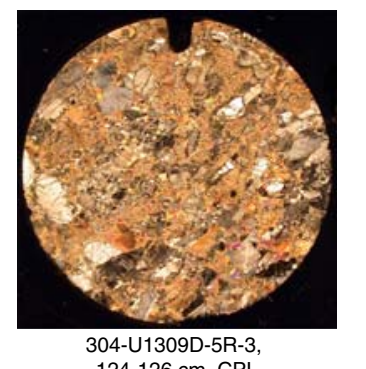

124-126 cm, CPL

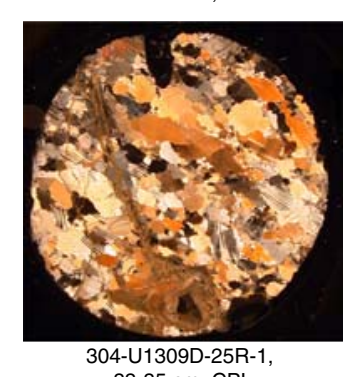

$33-35 \mathrm{~cm}, \mathrm{CPL}$

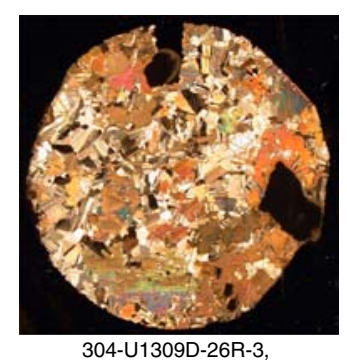

$83-85 \mathrm{~cm}, \mathrm{CPL}$

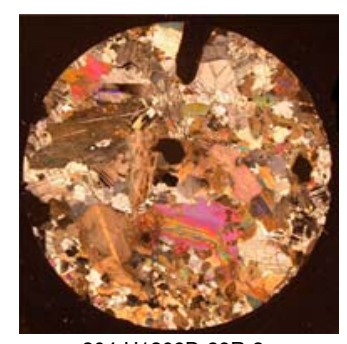

304-U1309D-29R-3, $64-66 \mathrm{~cm}, \mathrm{CPL}$

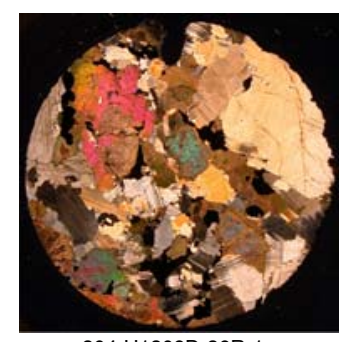

304-U1309D-36R-1 $10-12 \mathrm{~cm}, \mathrm{CPL}$
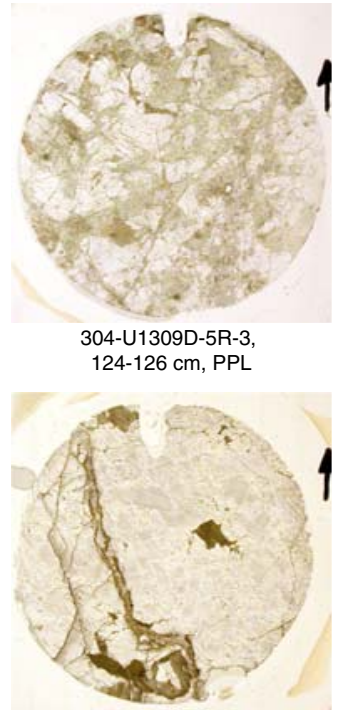

304-U1309D-25R-1, $33-35 \mathrm{~cm}, \mathrm{PPL}$
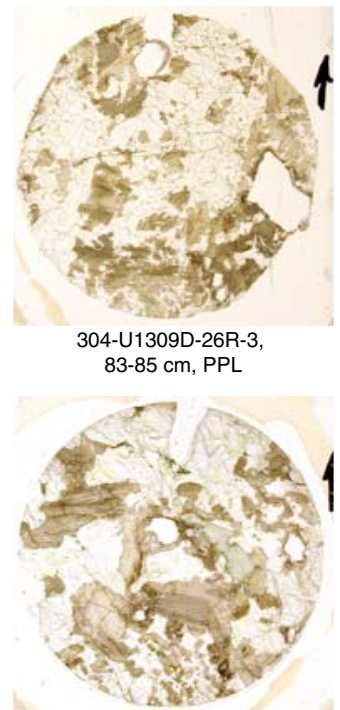

304-U1309D-29R-3, $64-66 \mathrm{~cm}, \mathrm{PPL}$

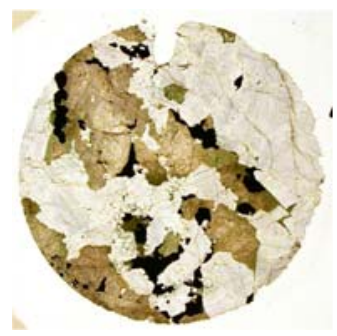

304-U1309D-36R-1, $10-12 \mathrm{~cm}, \mathrm{PPL}$

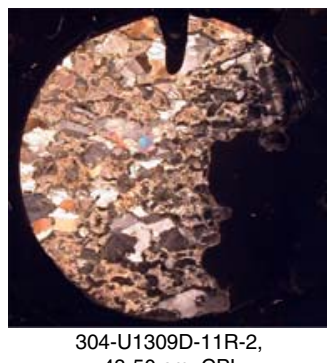

$48-50 \mathrm{~cm}, \mathrm{CPL}$

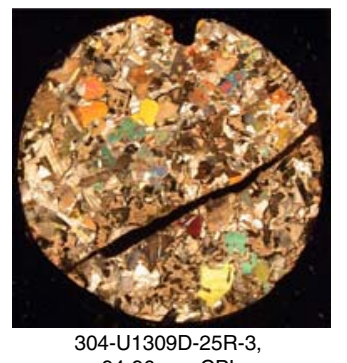

94-96 cm, CPL

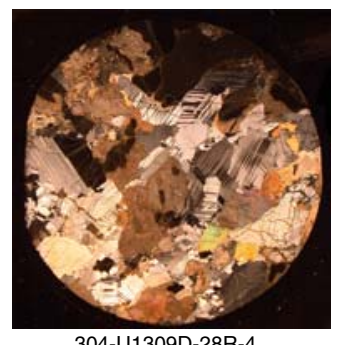

304-U1309D-28R-4, $45-47 \mathrm{~cm}, \mathrm{CPL}$

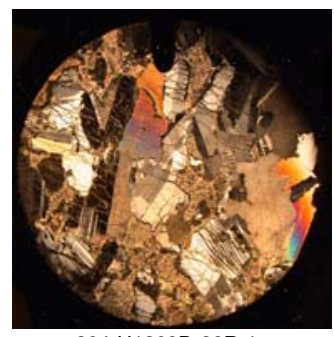

304-U1309D-32R-1, 9-11 cm, CPL

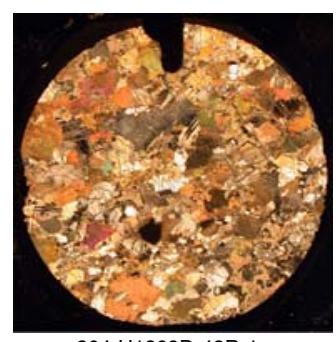

304-U1309D-43R-1, $57-79 \mathrm{~cm}, \mathrm{CPL}$
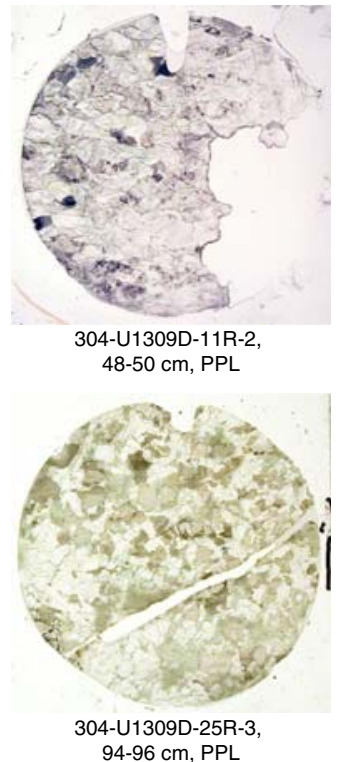

94-96 cm, PPL

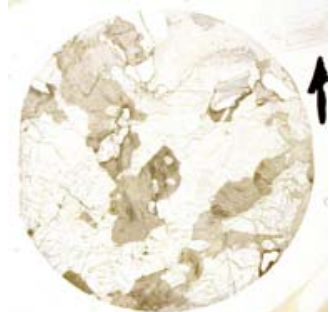

304-U1309D-28R-4 $45-47 \mathrm{~cm}, \mathrm{PPL}$

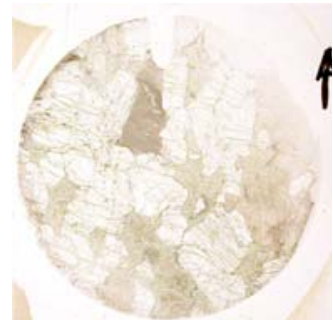

304-U1309D-32R-1 9-11 cm, PPL

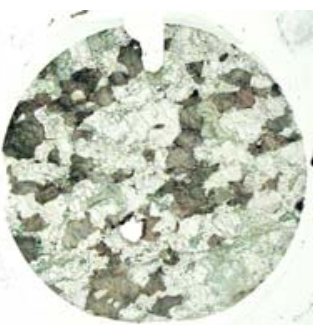

304-U1309D-43R-1, $57-79 \mathrm{~cm}, \mathrm{PPL}$ 
Plate P1 (continued). (Continued on next page.)

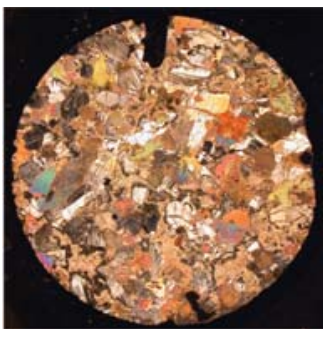

304-U1309D-44R-1

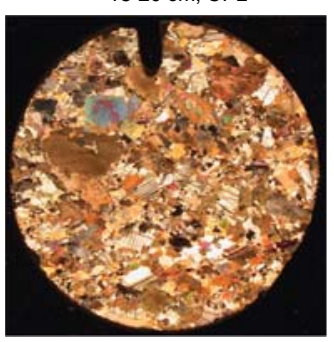

304-U1309D-51R-1

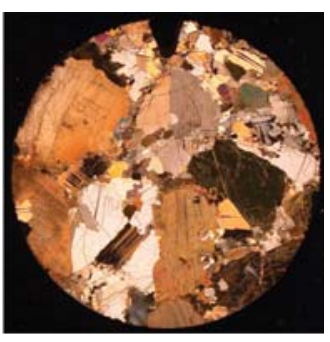

304-U1309D-58R-1
110-112 cm CPL

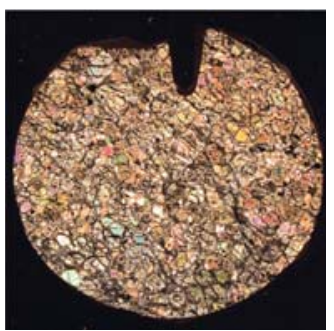

304-U1309D-63R-3, $41-43 \mathrm{~cm}, \mathrm{CPL}$

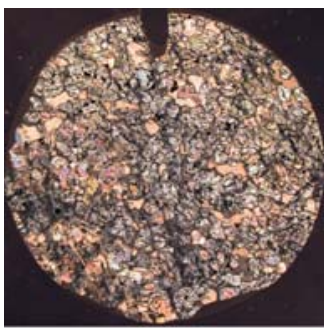

304-U1309D-66R-2, 93-95 cm, CPL

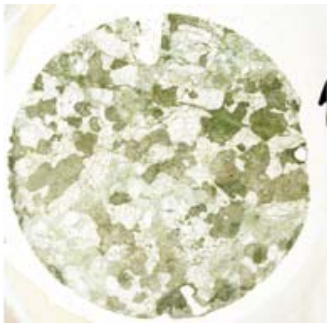

304-U1309D-44R-1, $18-20 \mathrm{~cm}, \mathrm{CPL}$

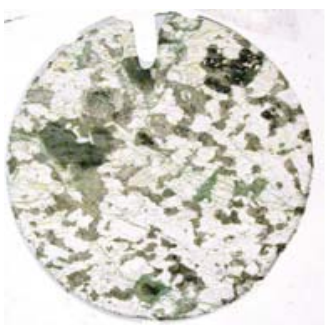

304-U1309D-51R-1, 74-76 cm, PPL

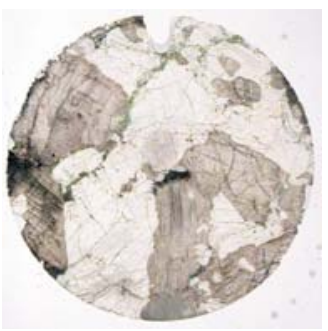

304-U1309D-58R-1, 110-112 cm, PPL

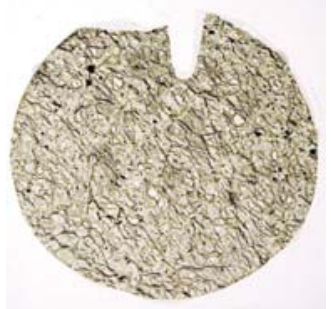

304-U1309D-63R-3, $41-43 \mathrm{~cm}, \mathrm{PPL}$

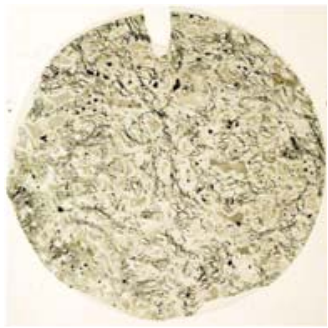

304-U1309D-66R-2, 93-95 cm, PPL

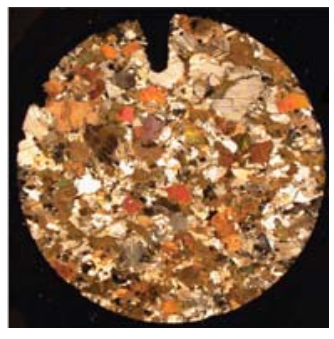

304-U1309D-44R-2

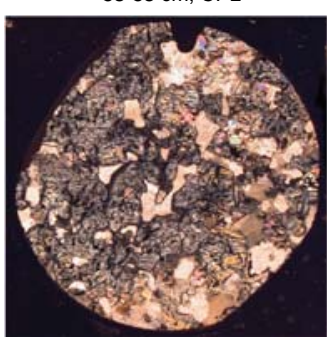

304-U1309D-54R-3, $45-47 \mathrm{~cm}, \mathrm{CPL}$

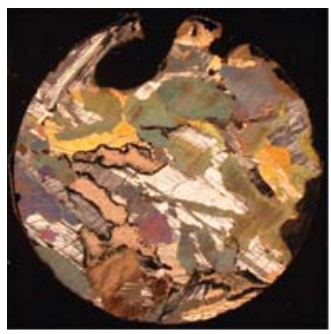

304-U1309D-59R-1

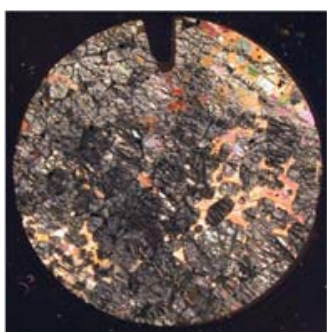

304-U1309D-64R-1, $58-60 \mathrm{~cm}, \mathrm{CPL}$

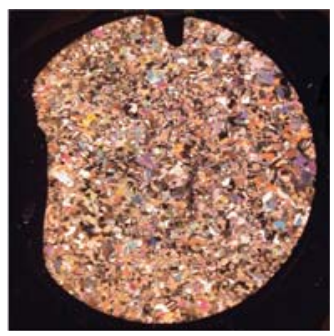

304-U1309D-67R-3,

$17-19 \mathrm{~cm}, \mathrm{CPL}$

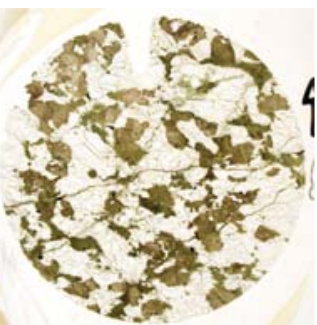

304-U1309D-44R-2, $83-85 \mathrm{~cm}, \mathrm{PPL}$

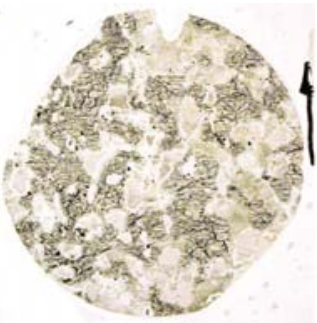

304-U1309D-54R-3, 45- $47 \mathrm{~cm}, \mathrm{PPL}$

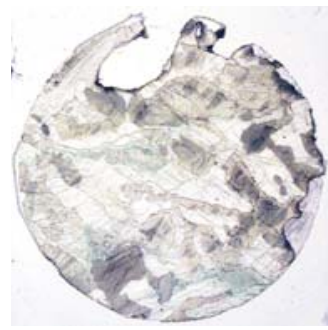

304-U1309D-59R-1, $58-60 \mathrm{~cm}, \mathrm{PPL}$

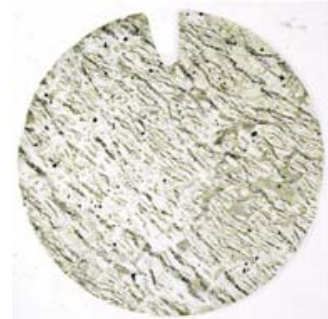

304-U1309D-64R-1, $58-60 \mathrm{~cm}, \mathrm{PPL}$

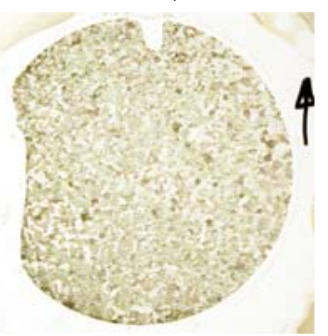

304-U1309D-67R-3, $17-19 \mathrm{~cm}, \mathrm{PPL}$ 
Plate P1 (continued). (Continued on next page.)

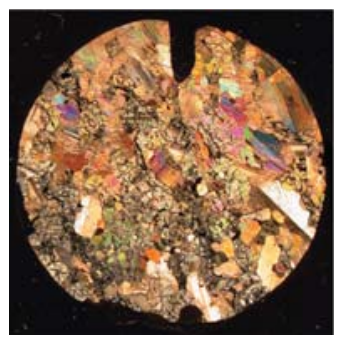

304-U1309D-70R-2,

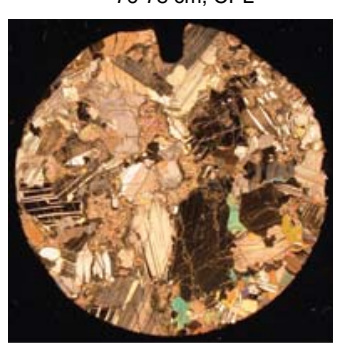

304-U1309D-71R-5

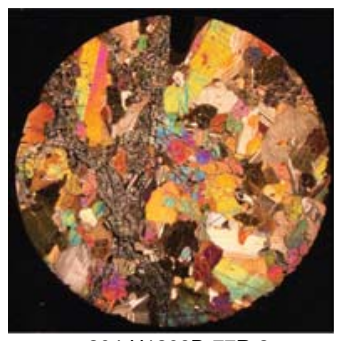

304-U1309D-77R-3,

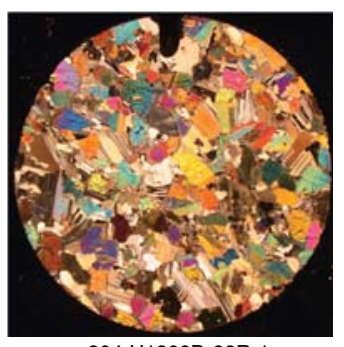

304-U1309D-88R-1 2-4 cm, CPL

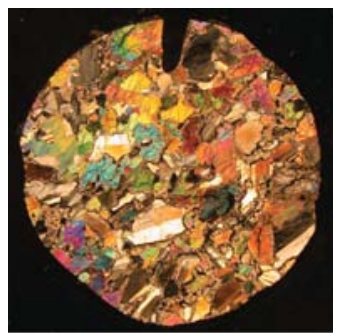

304-U1309D-90R-3, 8-10 cm, CPL

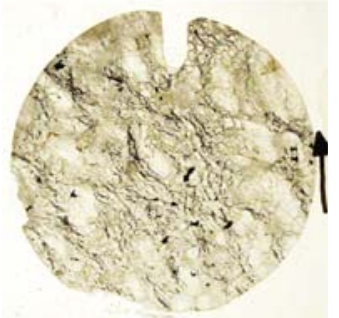

304-U1309D-70R-2,

$76-78 \mathrm{~cm}$, PPL

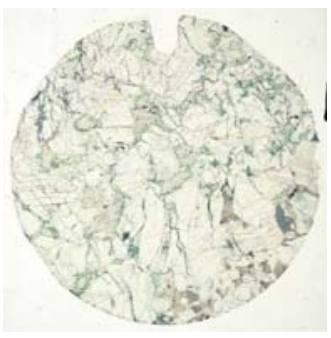

304-U1309D-71R-5,

13-15 cm, PPL

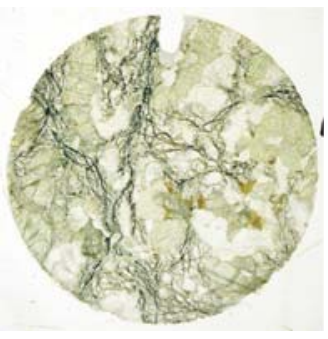

304-U1309D-77R-3

$21-23 \mathrm{~cm}, \mathrm{PPL}$

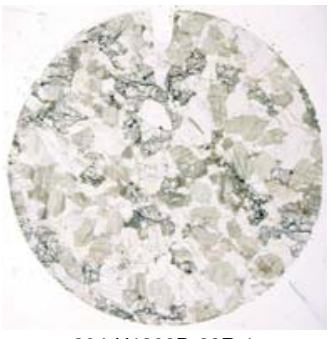

304-U1309D-88R-1, 2-4 cm, PPL

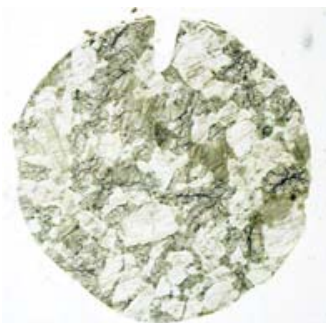

304-U1309D-90R-3, 8-10 cm, PPL

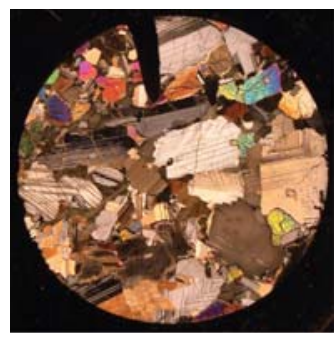

304-U1309D-71R-1,

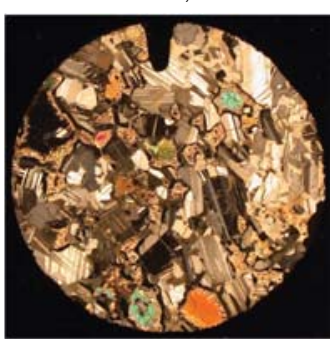

304-U1309D-73R-2,

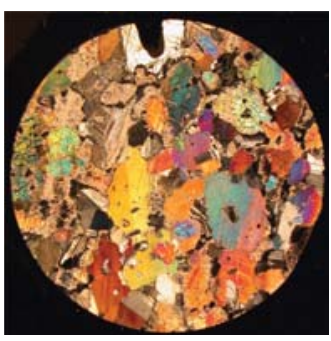

304-U1309D-83R-1,

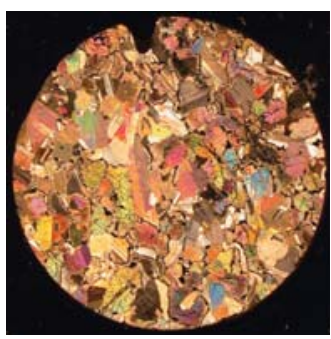

304-U1309D-89R-1

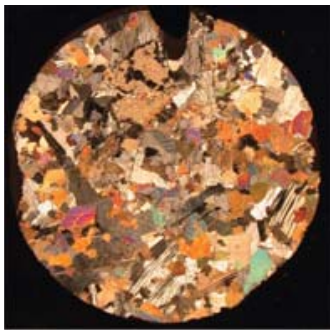

304-U1309D-92R-1,

$123-125 \mathrm{~cm}, \mathrm{CPL}$

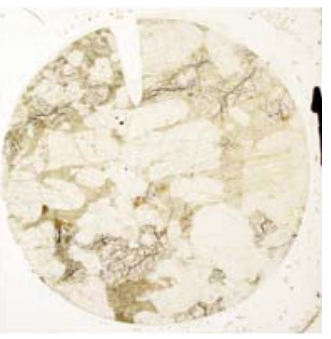

304-U1309D-71R-1,

$14-16 \mathrm{~cm}, \mathrm{PPL}$

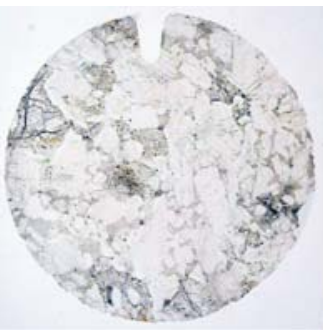

304-U1309D-73R-2

$86-88 \mathrm{~cm}, \mathrm{PPL}$

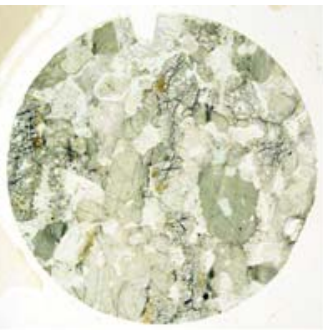

304-U1309D-83R-2, 29-31 cm, PPL

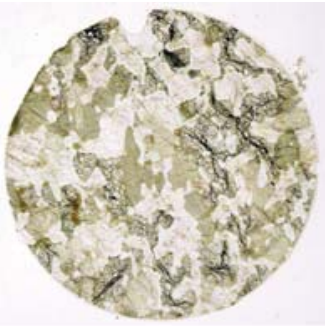

304-U1309D-89R-1, 92-94 cm, PPL

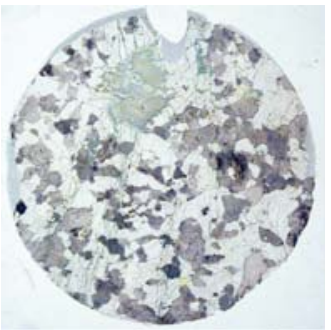

304-U1309D-92R-1, $123-125 \mathrm{~cm}, \mathrm{PPL}$ 
Plate P1 (continued). (Continued on next page.)

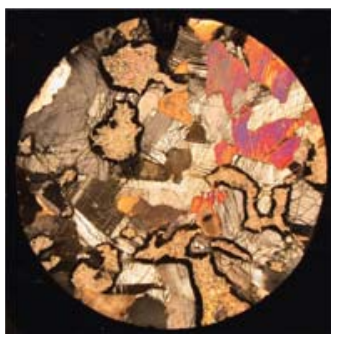

304-U1309D-95R-3

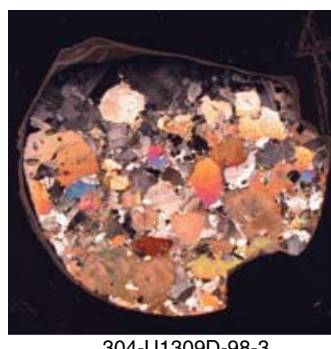

304-U1309D-98-3,

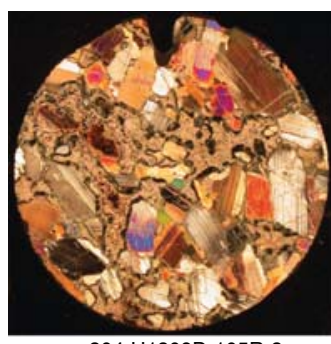

304-U1309D-105R-3

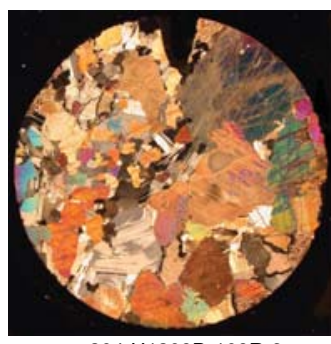

304-U1309D-109R-2,

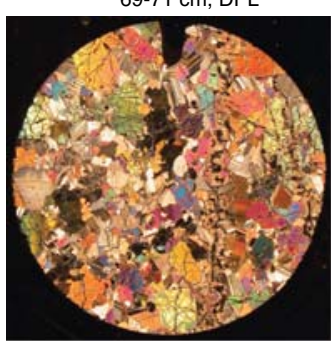

304-U1309D-111R-2, $17-19 \mathrm{~cm}, \mathrm{CPL}$

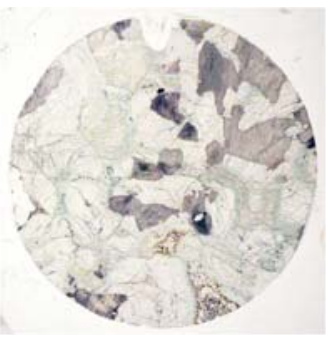

304-U1309D-95R-3,

$35-37 \mathrm{~cm}, \mathrm{PPL}$

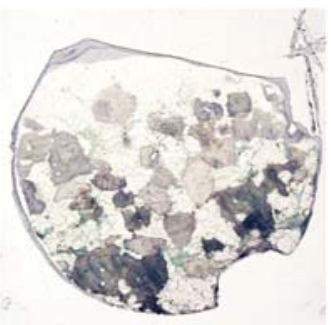

304-U1309D-98R-3,

96-98 cm, PPL

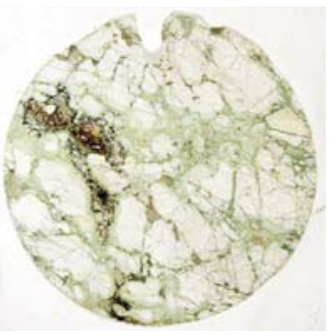

304-U1309D-105R-3

$46-48 \mathrm{~cm}, \mathrm{PPL}$

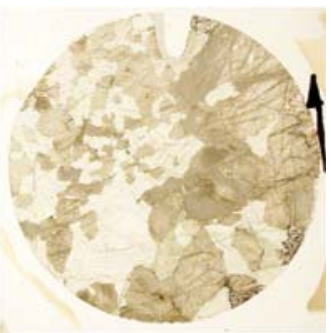

304-U1309D-109R-2, 69-71 cm, PPL

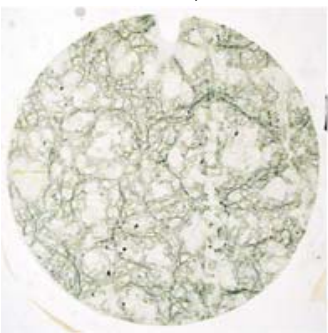

304-U1309D-111R-2, $17-19 \mathrm{~cm}, \mathrm{PPL}$

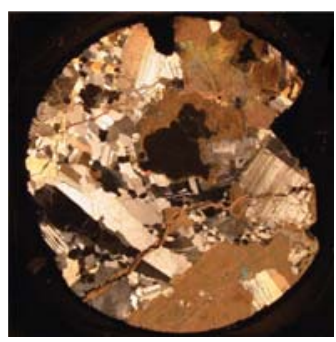

304-U1309D-97R-1

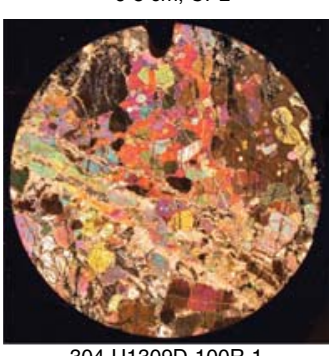

304-U1309D-100R-1,

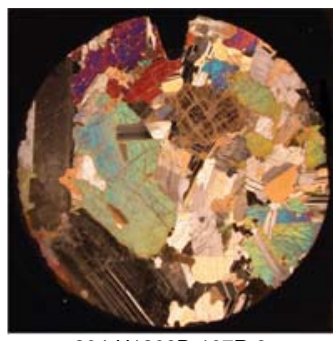

304-U1309D-107R-2,

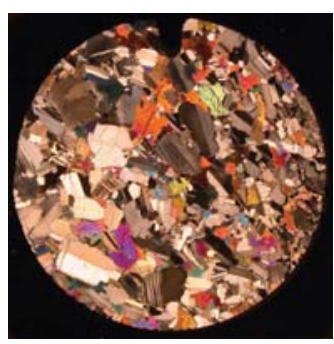

304-U1309D-110R-2,

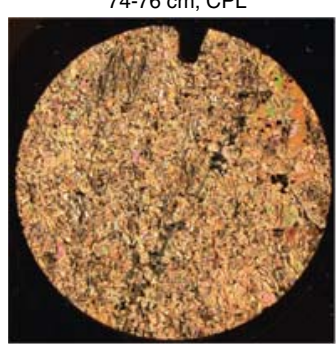

304-U1309D-111R-4,

$19-21 \mathrm{~cm}, \mathrm{CPL}$

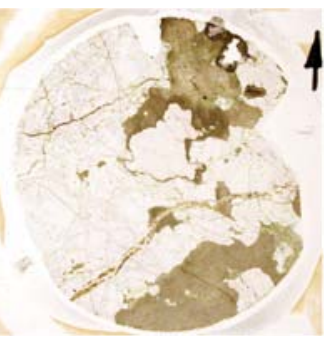

304-U1309D-97R-1,

6-8 cm, PPL

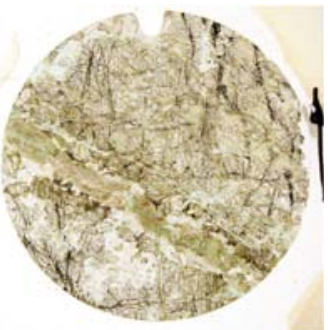

304-U1309D-100R-1,

$32-34 \mathrm{~cm}, \mathrm{PPL}$

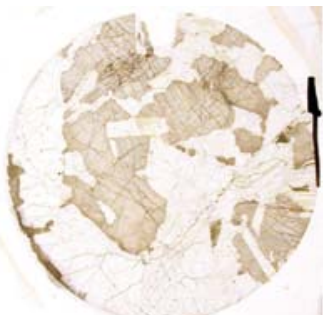

304-U1309D-107R-2, $52-54 \mathrm{~cm}, \mathrm{PPL}$

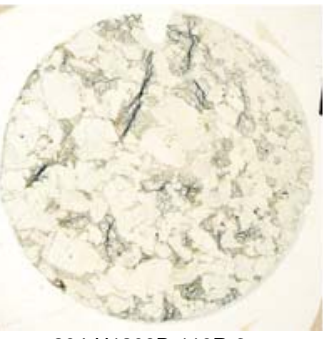

304-U1309D-110R-2, $74-76 \mathrm{~cm}, \mathrm{PPL}$

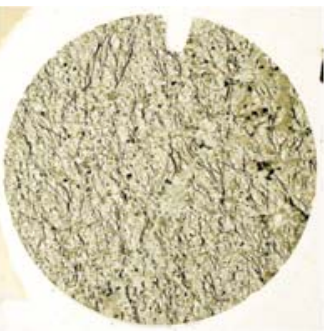

304-U1309D-111R-4, $19-21 \mathrm{~cm}, \mathrm{PPL}$ 
Plate P1 (continued). (Continued on next page.)

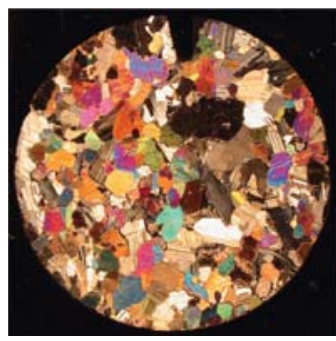

304-U1309D-117R-1

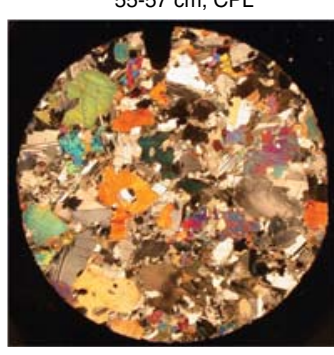

304-U1309D-120R-2,

$26-28 \mathrm{~cm}, \mathrm{CPL}$

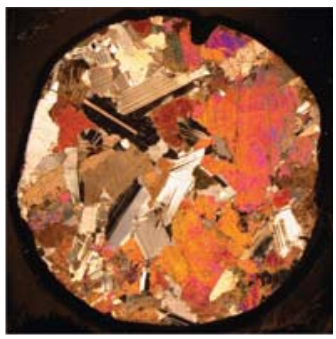

304-U1309D-127R-2,

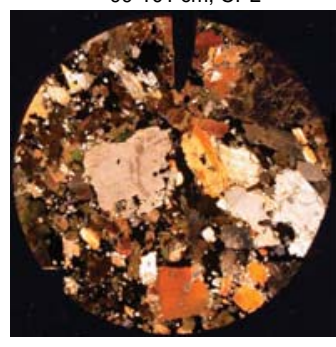

304-U1309D-130R-1,

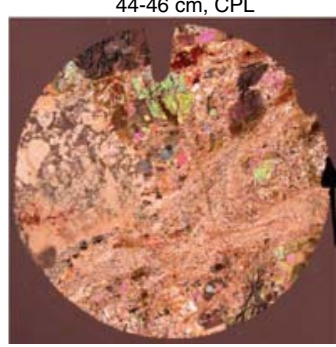

304-U1309D-136R-1, $32-34 \mathrm{~cm}, \mathrm{CPL}$

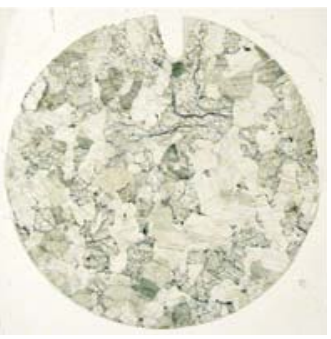

304-U1309D-117R-2, $55-57 \mathrm{~cm}, \mathrm{PPL}$

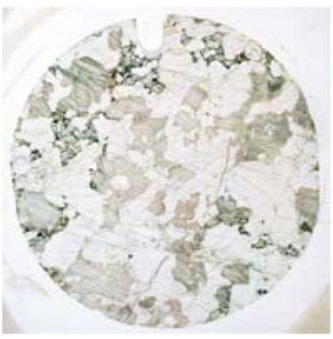

304-U1309D-120R-2, $26-28 \mathrm{~cm}, \mathrm{PPL}$

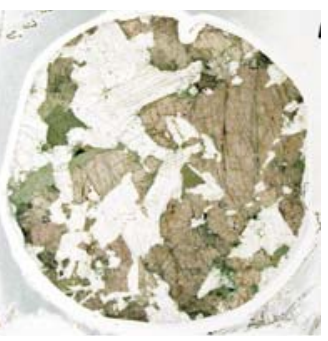

304-U1309D-127R-2,

99-101 cm, PPL

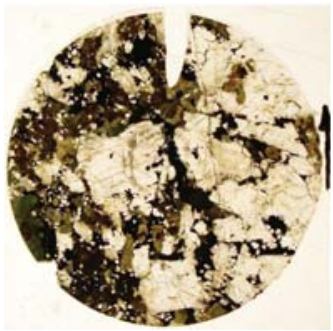

304-U1309D-130R-1,

$44-46 \mathrm{~cm}$, PPL

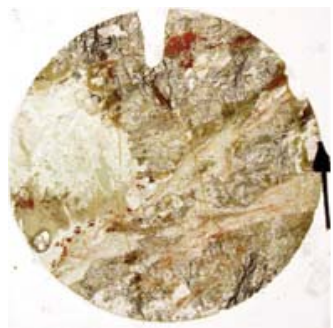

304-U1309D-136R-1, $32-34 \mathrm{~cm}, \mathrm{PPL}$

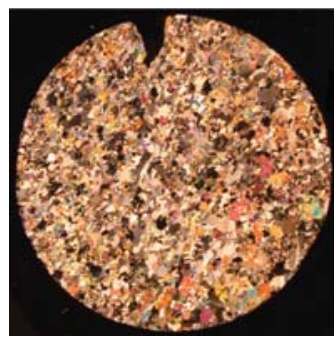

304-U1309D-117R-4 $27-29 \mathrm{~cm}, \mathrm{CPL}$

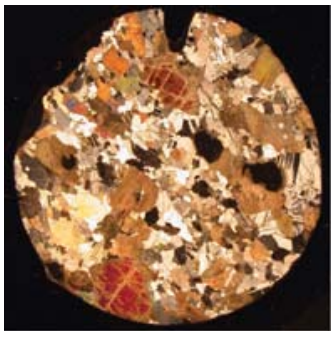

304-U1309D-124R-4 $47-49 \mathrm{~cm}, \mathrm{CPL}$

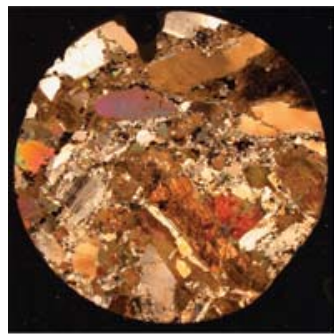

304-U1309D-128R-3

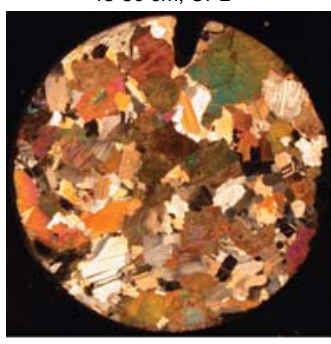

304-U1309D-132R-3,

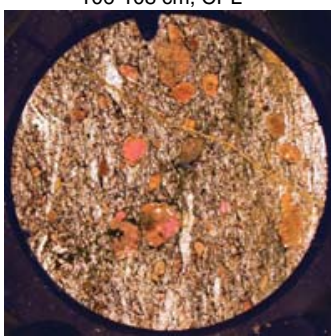

304-U1309D-137R-2,

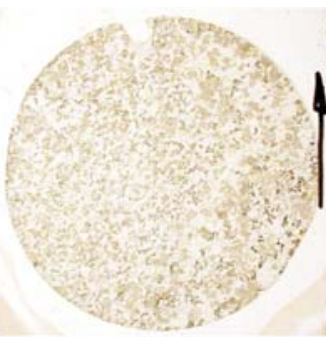

304-U1309D-117R-4

27-29 cm, PPL

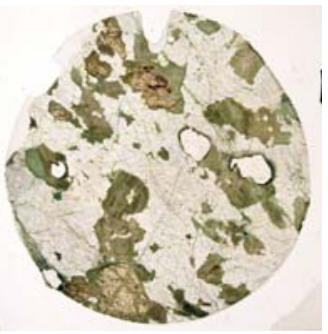

304-U1309D-124R-4,

$47-49 \mathrm{~cm}, \mathrm{PPL}$

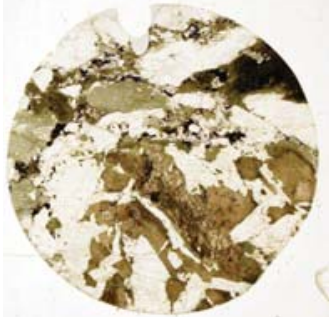

304-U1309D-128R-3,

48-50 cm, PPL

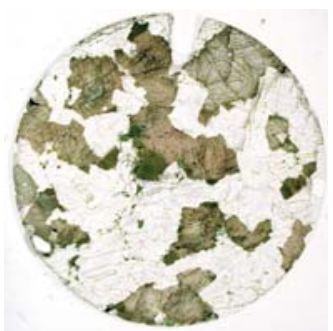

304-U1309D-132R-3,

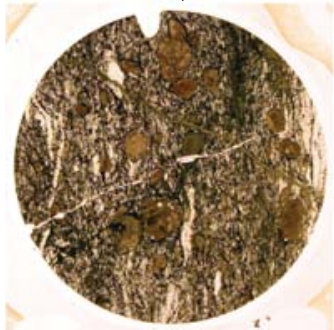

304-U1309D-137R-2, $126-128 \mathrm{~cm}, \mathrm{PPL}$ 
Plate P1 (continued). (Continued on next page.)

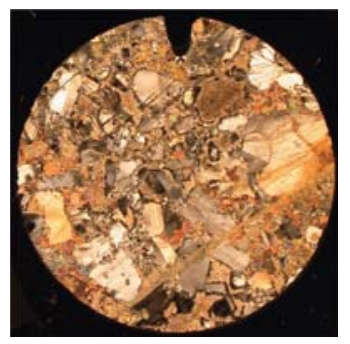

304-U1309D-141R-2,

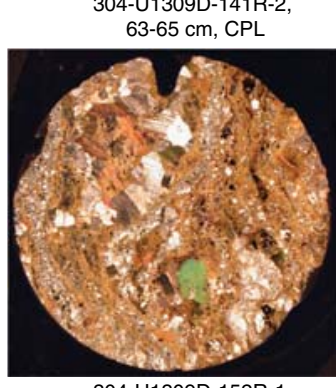

304-U1309D-152R-

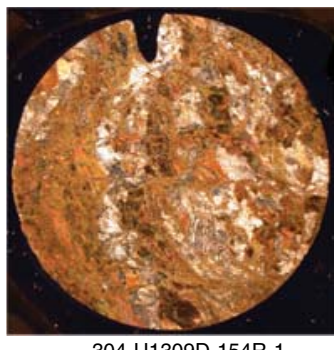

304-U1309D-154R-1

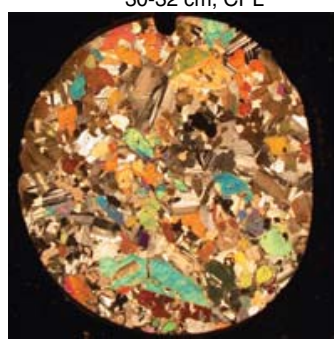

304-U1309D-159R-1,

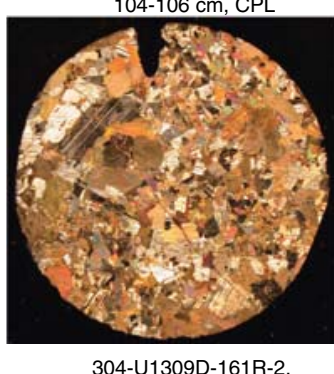

$44-46 \mathrm{~cm}, \mathrm{CPL}$

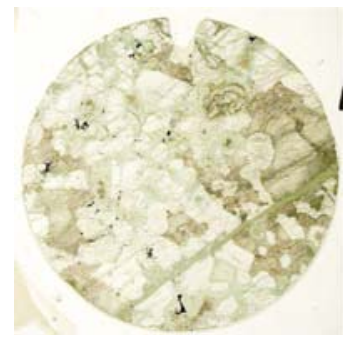

304-U1309D-141R-2, $63-65 \mathrm{~cm}, \mathrm{PPL}$

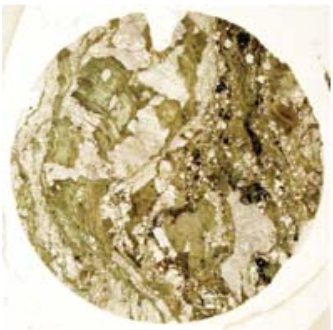

304-U1309D-152R-1,

$35-37 \mathrm{~cm}, \mathrm{PPL}$

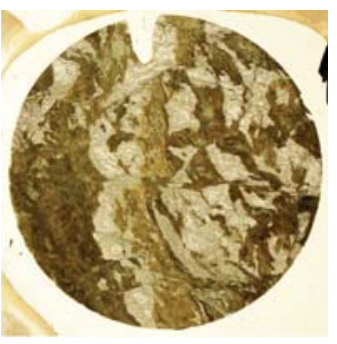

304-U1309D-154R-1, 30-32 cm, PPL

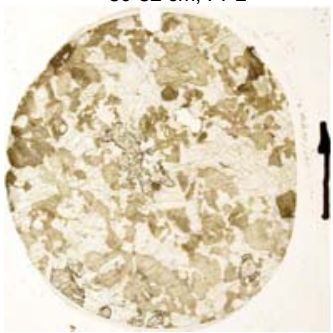

304-U1309D-159R-1,

104-106 cm, PPL

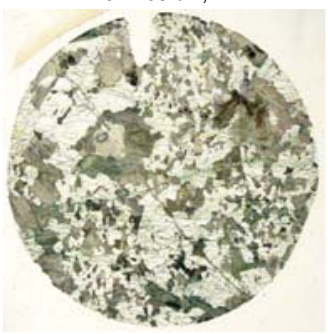

304-U1309D-161R-2,

44-46 cm, PPL

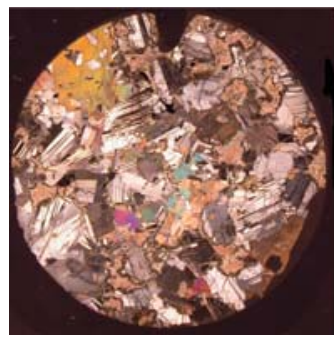

304-U1309D-145R-3,

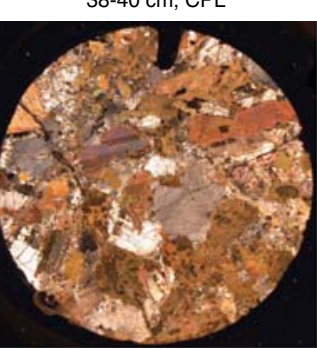

304-U1309D-153R-2

$88-90 \mathrm{~cm}, \mathrm{CPL}$

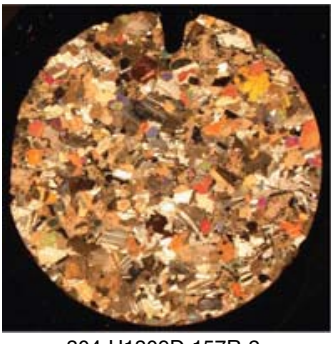

304-U1309D-157R-2

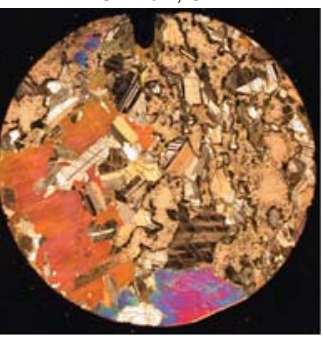

304-U1309D-160R-2,

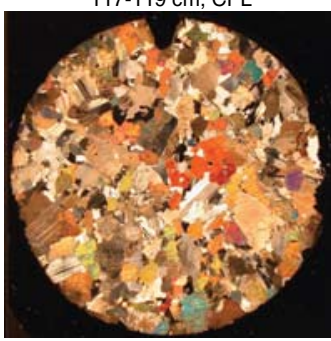

304-U1309D-164R-3,

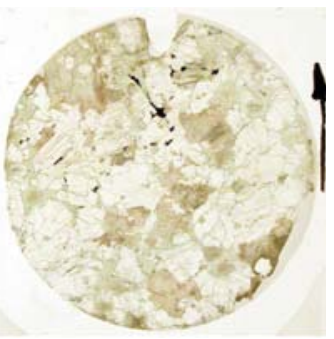

304-U1309D-145R-3 $38-40 \mathrm{~cm}, \mathrm{PPL}$

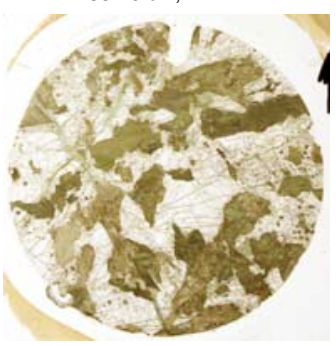

304-U1309D-153R-2

$88-90 \mathrm{~cm}, \mathrm{PPL}$

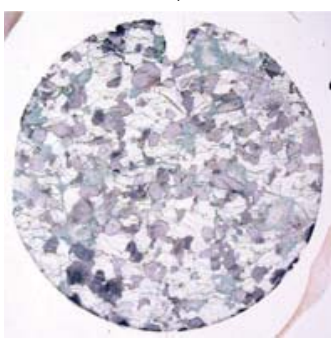

304-U1309D-157R-2

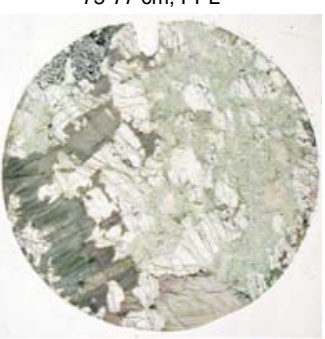

304-U1309D-160R-2

$117-119 \mathrm{~cm}, \mathrm{PPL}$

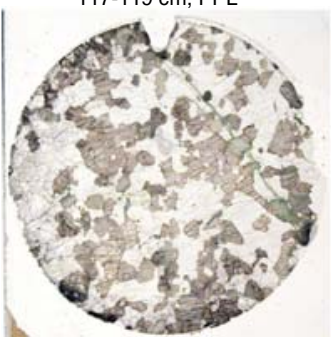

304-U1309D-164R-3,

110-112 cm, PPL 
Plate P1 (continued). (Continued on next page.)

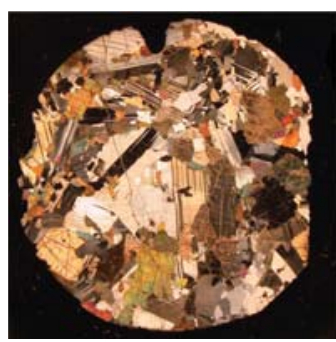

304-U1309D-169R-1,

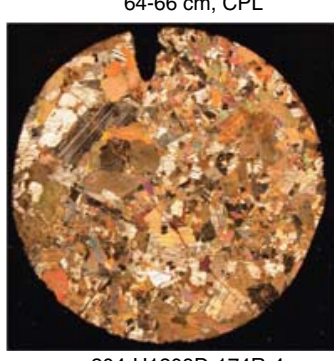

304-U1309D-174R-4

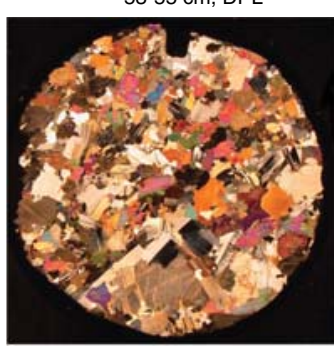

304-U1309D-188R-2,

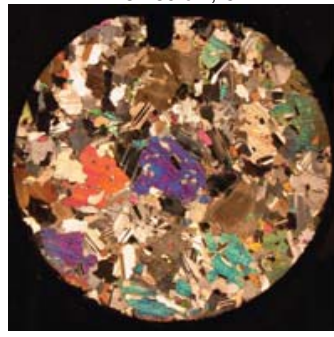

304-U1309D-190R-1 $31-33 \mathrm{~cm}, \mathrm{CPL}$

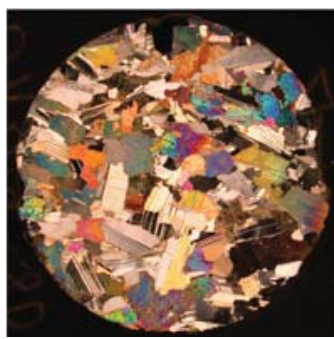

304-U1309D-200R-2, $31-33 \mathrm{~cm}, \mathrm{CPL}$

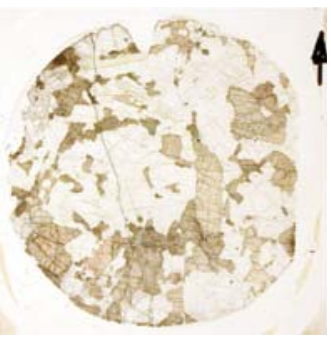

304-U1309D-169R-1, 64-66 cm, PPL

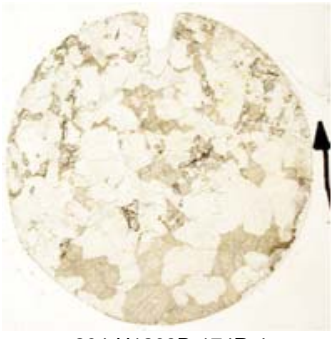

304-U1309D-174R-4 $53-55 \mathrm{~cm}, \mathrm{PPL}$

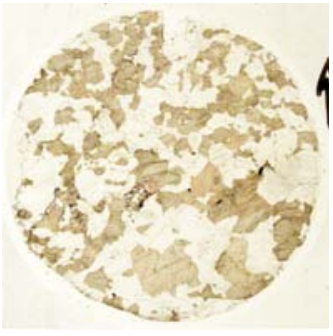

304-U1309D-188R-2 $37-39 \mathrm{~cm}, \mathrm{PPL}$

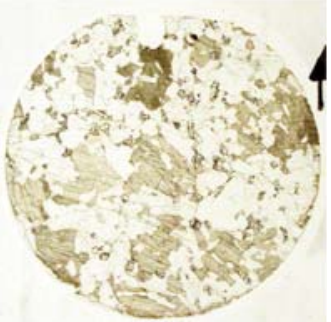

304-U1309D-190R-1, $31-33 \mathrm{~cm}, \mathrm{PPL}$

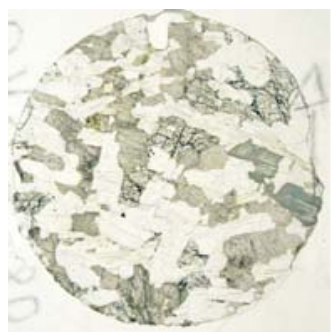

304-U1309D-200R-2 $31-33 \mathrm{~cm}, \mathrm{PPL}$

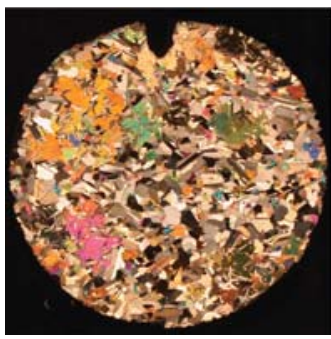

304-U1309D-170R-4

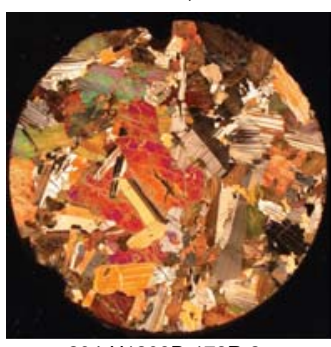

304-U1309D-178R-3,

8-10 cm, CPL

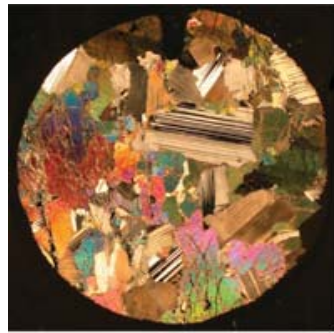

304-U1309D-189R-3,

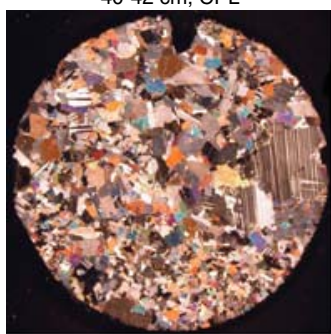

304-U1309D-194R-2 27-29 cm, CPL

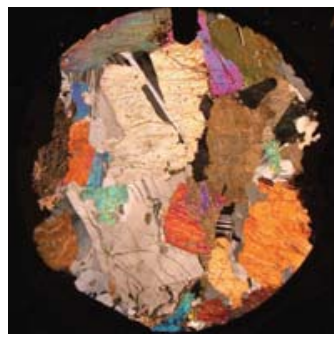

304-U1309D-202R-1,

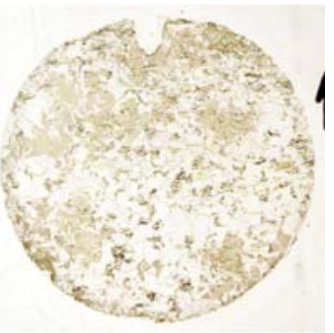

304-U1309D-170R-4 $77-79 \mathrm{~cm}, \mathrm{PPL}$

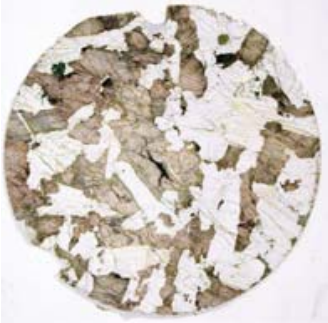

304-U1309D-178R-3,

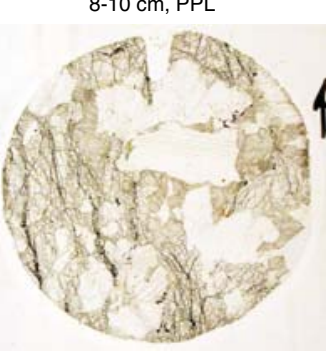

304-U1309D-189R-3, 40-42 cm, PPL

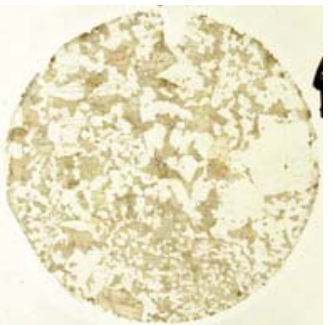

304-U1309D-194R-2 $27-29 \mathrm{~cm}, \mathrm{PPL}$

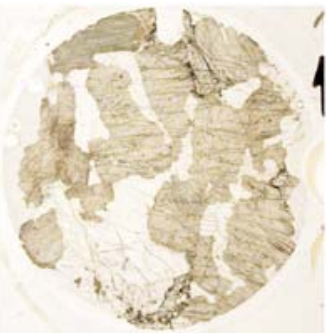

304-U1309D-202R-1, 
Plate P1 (continued). (Continued on next page.)
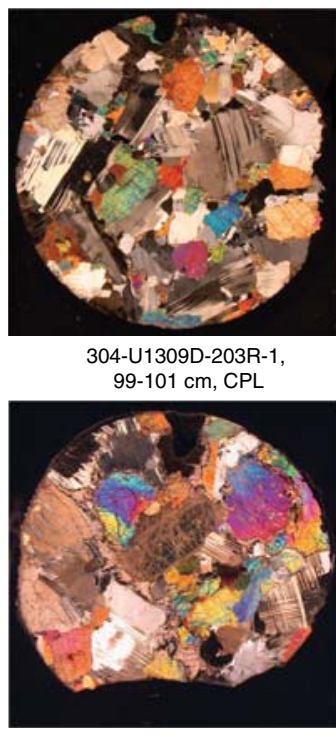

304-U1309D-205R-2

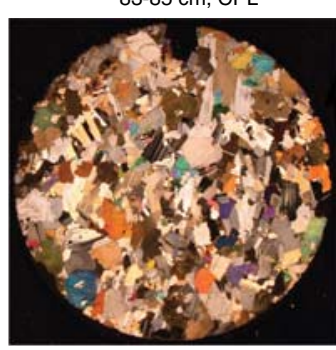

304-U1309D-211R-1,

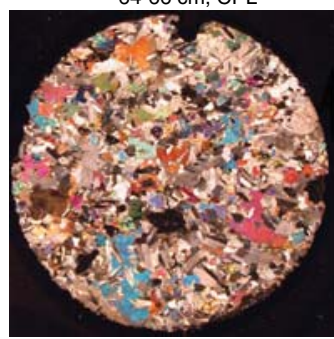

304-U1309D-216R-1

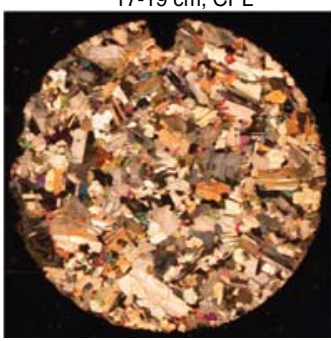

304-U1309D-221R-3 $64-66 \mathrm{~cm}, \mathrm{CPL}$

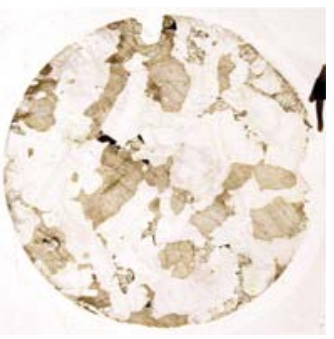

304-U1309D-203R-1 99-101 cm, PPL

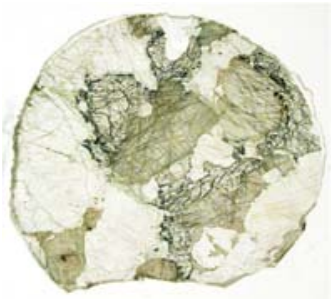

304-U1309D-205R-2 $83-85 \mathrm{~cm}, \mathrm{PPL}$

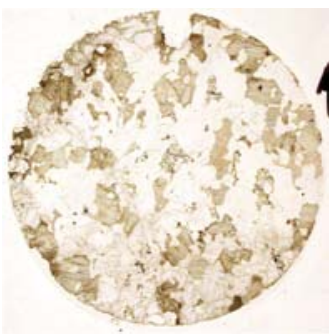

304-U1309D-211R-1, 64-66 cm, PPL

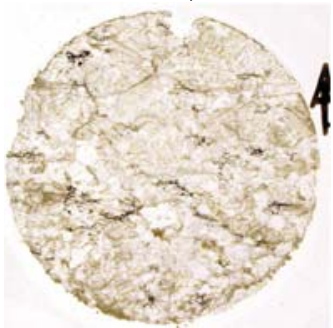

304-U1309D-216R-1, $17-19 \mathrm{~cm}, \mathrm{PPL}$

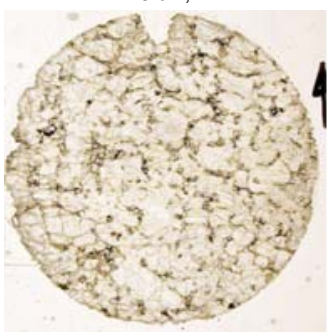

304-U1309D-221R-3, 64-66 cm, PPL

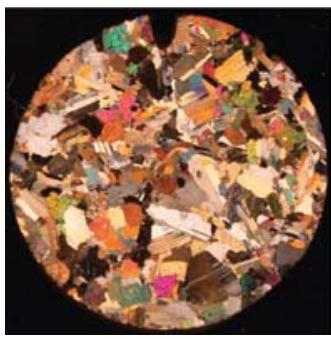

304-U1309D-204R-2

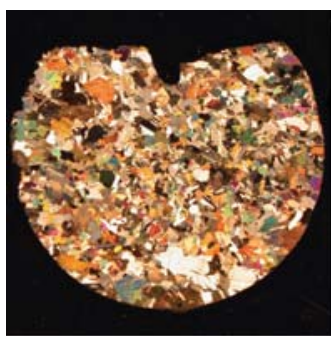

304-U1309D-208R-2

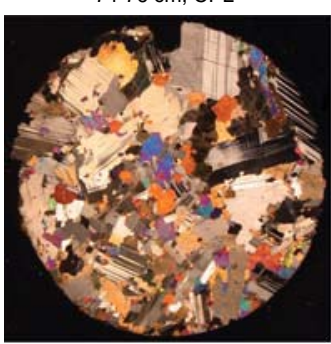

304-U1309D-212R-4

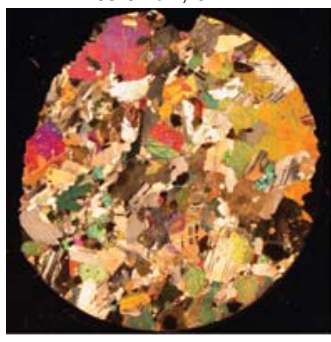

304-U1309D-217R-2,

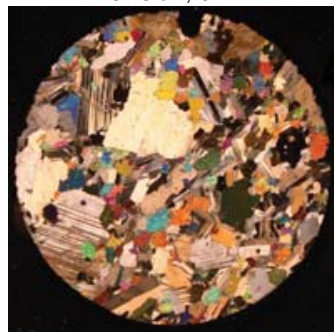

304-U1309D-225R-1,

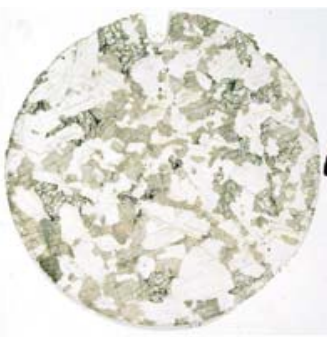

304-U1309D-204R-2, $97-99 \mathrm{~cm}, \mathrm{PPL}$

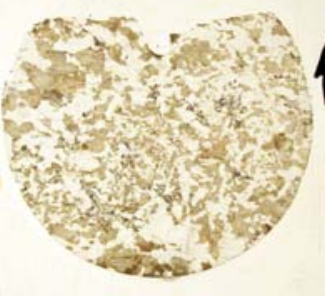

304-U1309D-208R-2 $74-76 \mathrm{~cm}, \mathrm{PPL}$

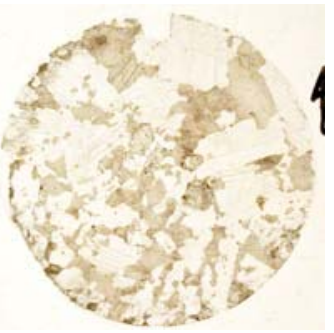

304-U1309D-212R-4

$65-67 \mathrm{~cm}, \mathrm{PPL}$

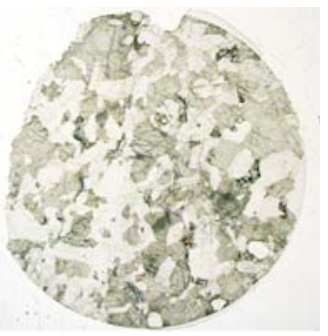

304-U1309D-217R-2 23- $25 \mathrm{~cm}, \mathrm{PPL}$

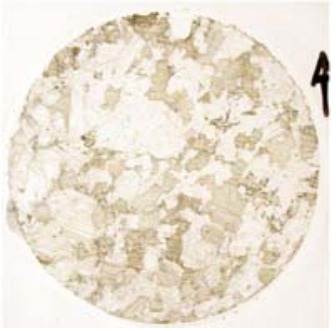

304-U1309D-225R-1, $72-74 \mathrm{~cm}, \mathrm{PPL}$ 
Plate P1 (continued). (Continued on next page.)
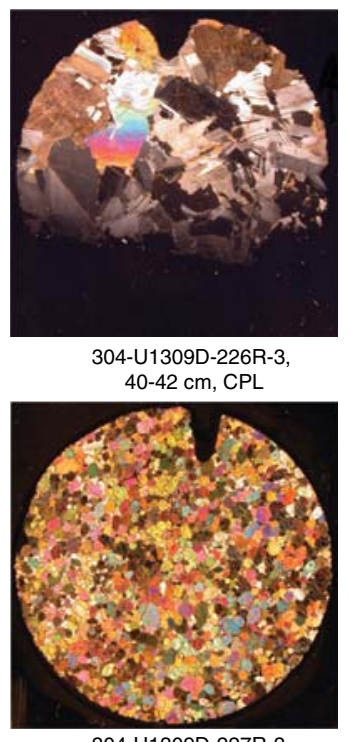

304-U1309D-227R-3,

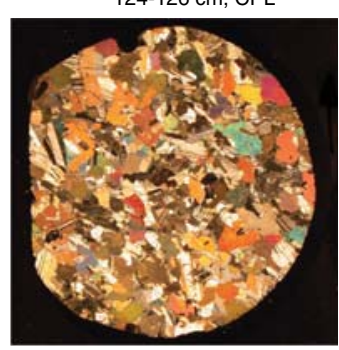

304-U1309D-230R-2

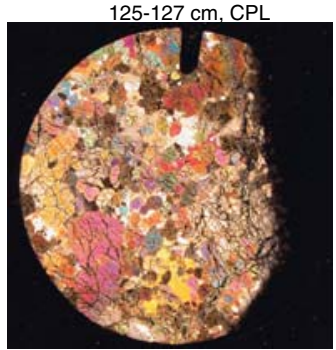

304-U1309D-232R-1,

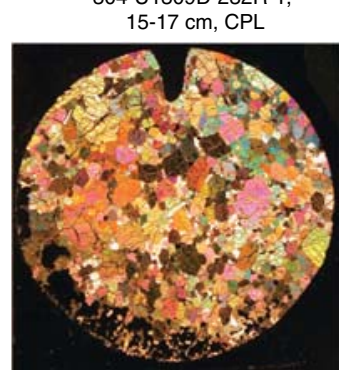

304-U1309D-234R-1, $27-29 \mathrm{~cm}, \mathrm{CPL}$

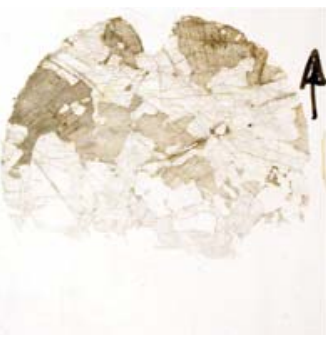

304-U1309D-226R-3, 40-42 cm, PPL

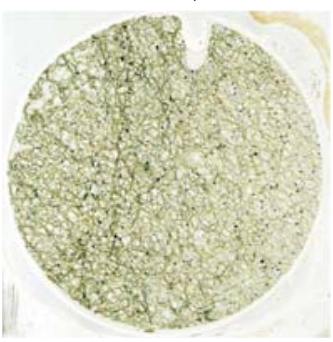

304-U1309D-227R-3,

$124-126 \mathrm{~cm}, \mathrm{PPL}$

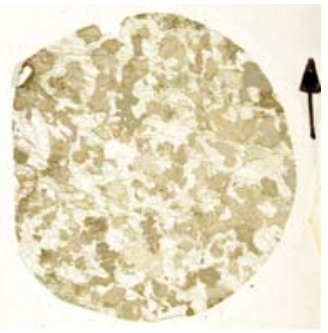

304-U1309D-230R-2,

$125-127 \mathrm{~cm}$, PP

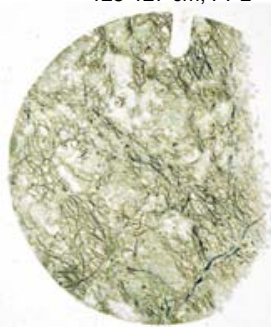

304-U1309D-232R-1 $15-17 \mathrm{~cm}, \mathrm{PPL}$

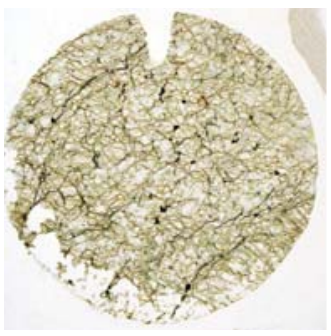

304-U1309D-234R-1, $27-29 \mathrm{~cm}, \mathrm{PPL}$

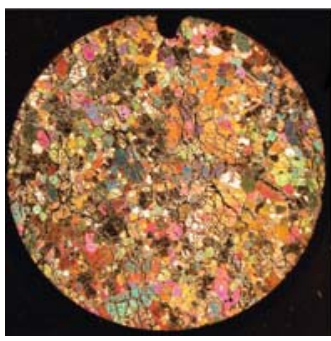

304-U1309D-227R-2

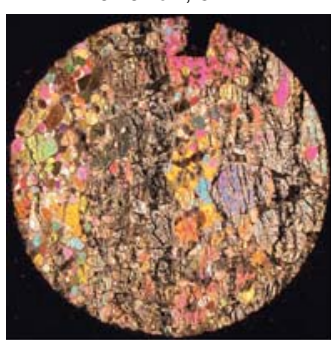

304-U1309D-228R-3,

29-31 cm, CPL

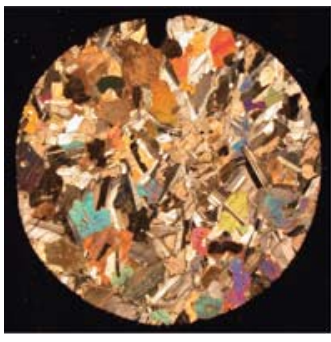

304-U1309D-230R-3,

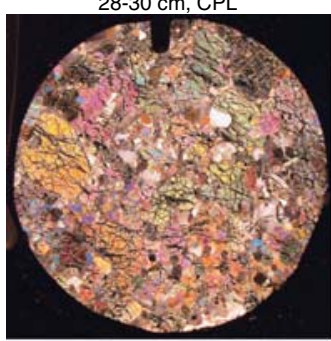

304-U1309D-233R-1

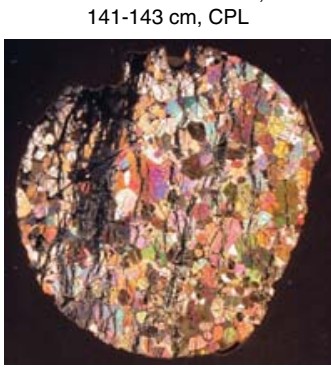

304-U1309D-235R-2,

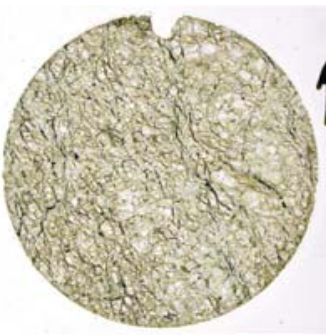

304-U1309D-227R-2, $82-84 \mathrm{~cm}, \mathrm{PPL}$

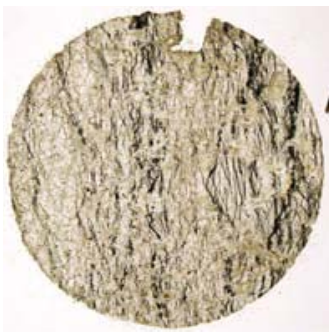

304-U1309D-228R-3,

29-31 cm, PPL

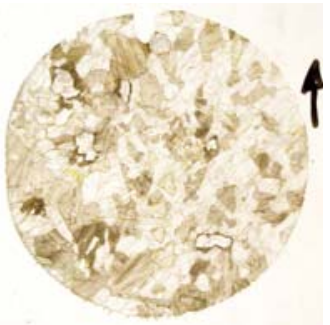

304-U1309D-230R-3, 28-30 cm, PPL

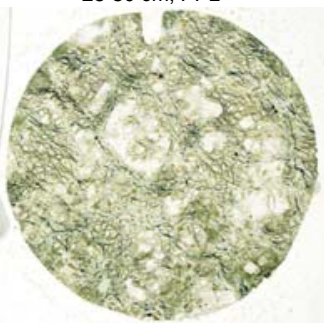

304-U1309D-233R-1, 141-143 cm, PPL

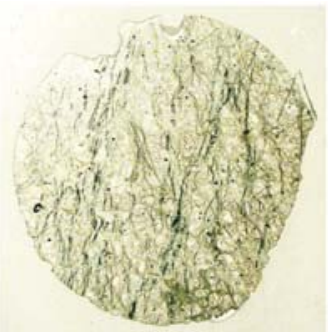

304-U1309D-235R-2, 
Plate P1 (continued). (Continued on next page.)

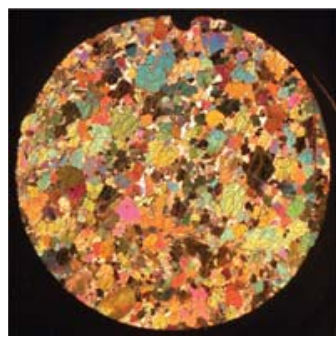

304-U1309D-236R-2, $125-127 \mathrm{~cm}, \mathrm{CPL}$

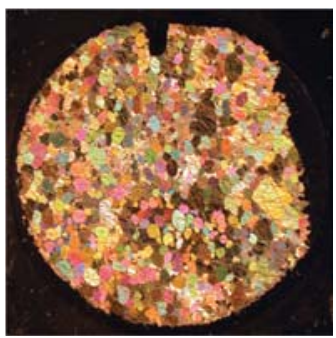

304-U1309D-241R-2

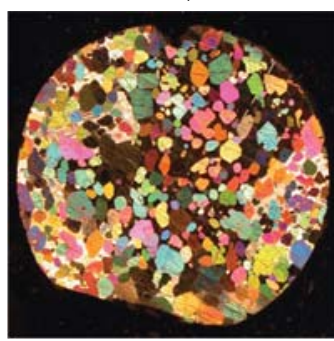

304-U1309D-247R-3

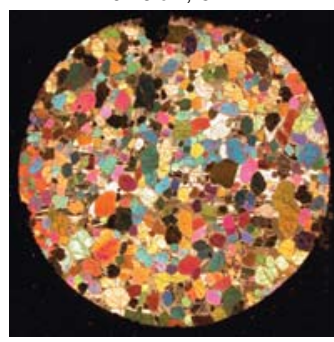

304-U1309D-248R-3

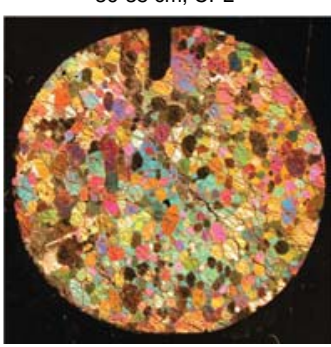

304-U1309D-256R-2 $83-85 \mathrm{~cm}, \mathrm{CPL}$

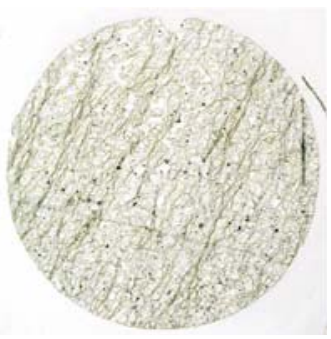

304-U1309D-236R-2, $125-127 \mathrm{~cm}$, PPL

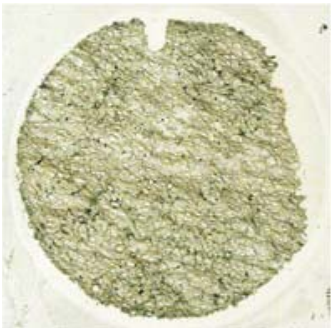

304-U1309D-241R-2,

89-91 cm, PPL

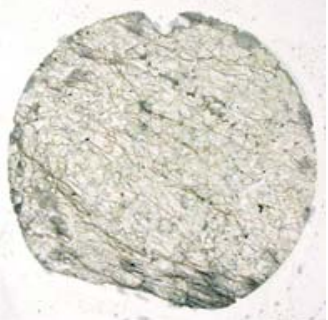

304-U1309D-247R-3,

$16-18 \mathrm{~cm}, \mathrm{PPL}$

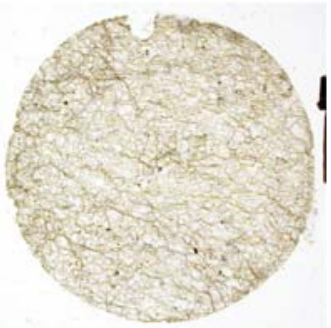

304-U1309D-248R-3,

$36-38 \mathrm{~cm}, \mathrm{PPL}$

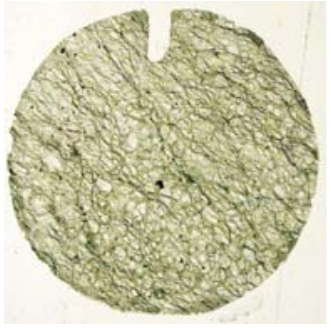

304-U1309D-256R-2, $83-85 \mathrm{~cm}, \mathrm{PPL}$

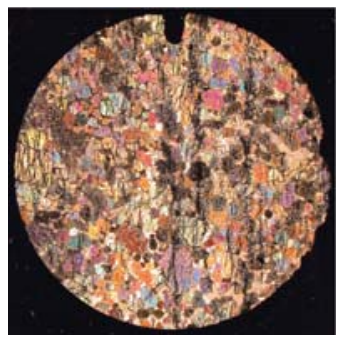

304-U1309D-240R-2

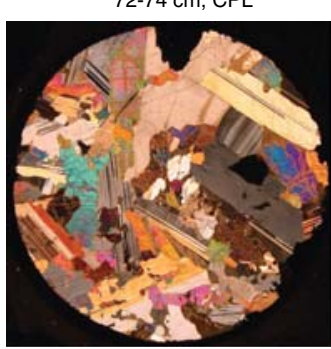

304-U1309D-244R-1

$34-36 \mathrm{~cm}, \mathrm{CPL}$

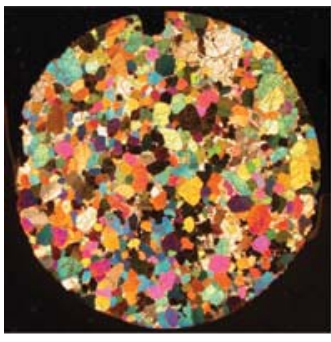

304-U1309D-248R-2,

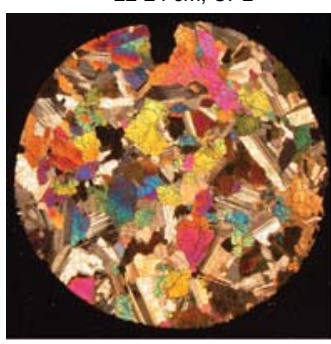

304-U1309D-251R-1

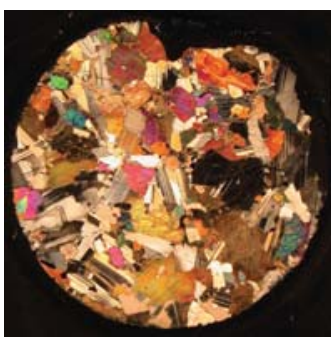

304-U1309D-262R-3, $112-114 \mathrm{~cm}, \mathrm{CPL}$

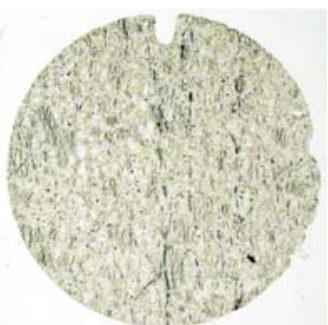

304-U1309D-240R-2,

$72-74 \mathrm{~cm}$, PPL

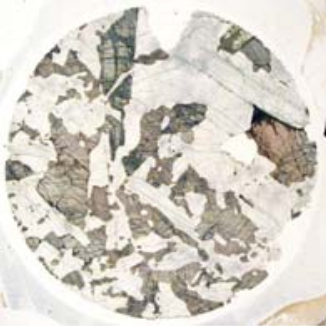

304-U1309D-244R-1,

$34-36 \mathrm{~cm}, \mathrm{PPL}$

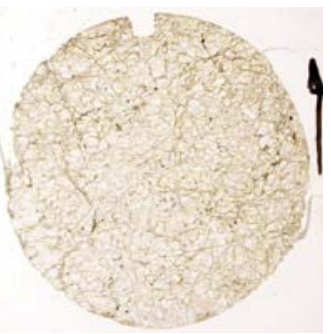

304-U1309D-248R-2,

$22-24 \mathrm{~cm}, \mathrm{PPL}$

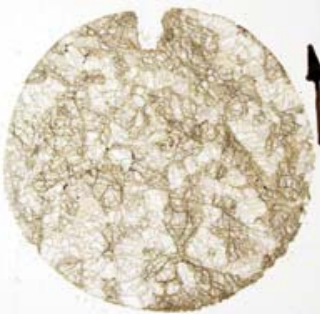

304-U1309D-251R-1, $87-89 \mathrm{~cm}, \mathrm{PPL}$

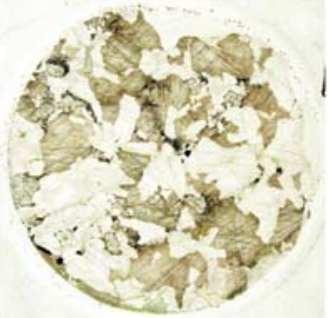

304-U1309D-262R-3, $112-114 \mathrm{~cm}, \mathrm{PPL}$ 


\section{Plate P1 (continued).}

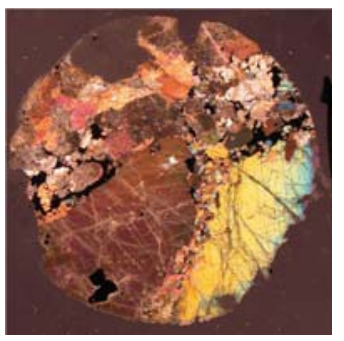

304-U1309D-264R-1, $81-83 \mathrm{~cm}, \mathrm{CPL}$

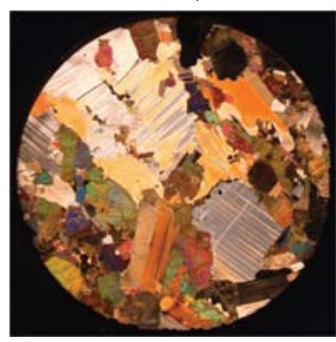

304-U1309D-271R-3, $59-61 \mathrm{~cm}, \mathrm{CPL}$

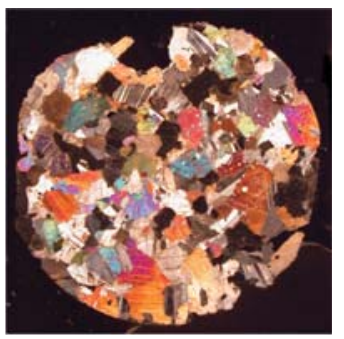

304-U1309D-280R-1,

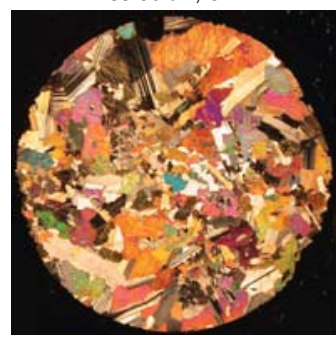

304-U1309D-290R-2, $89-91 \mathrm{~cm}, \mathrm{CPL}$

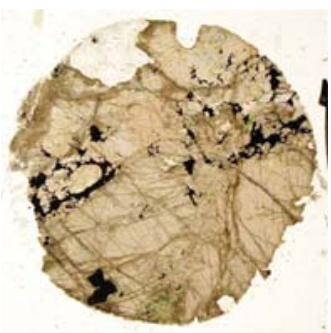

304-U1309D-164R-1, $81-83 \mathrm{~cm}, \mathrm{PPL}$

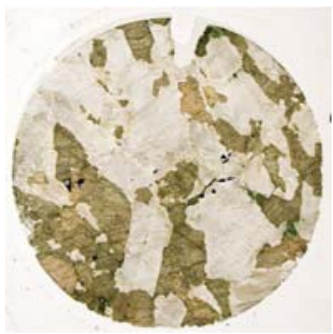

304-U1309D-271R-3, $59-61 \mathrm{~cm}, \mathrm{PPL}$

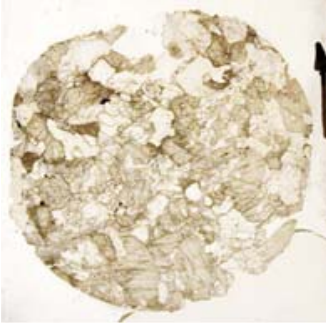

304-U1309D-280R-1, $88-90 \mathrm{~cm}, \mathrm{PP}$

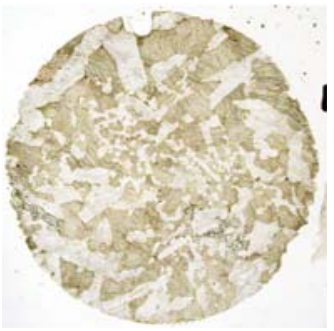

304-U1309D-290R-2, 89-91 cm, PPL

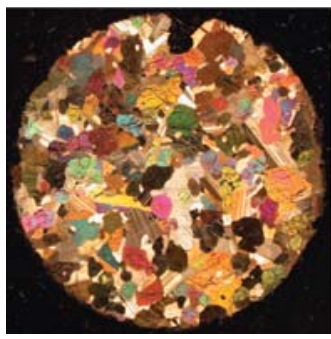

304-U1309D-268R-2,

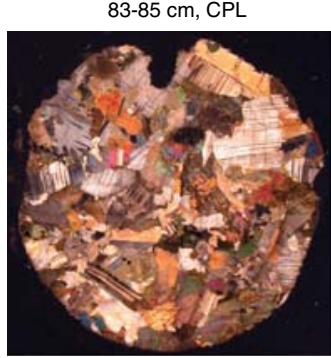

304-U1309D-275R-3, $88-90 \mathrm{~cm}, \mathrm{CPL}$

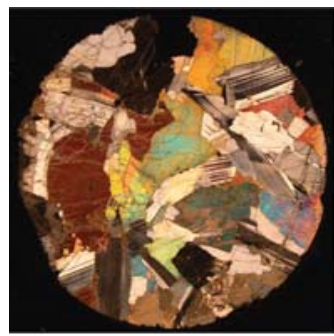

304-U1309D-285R-1,

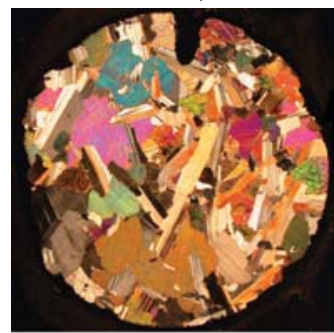

304-U1309D-291R-3,

$39-41 \mathrm{~cm}, \mathrm{CPL}$

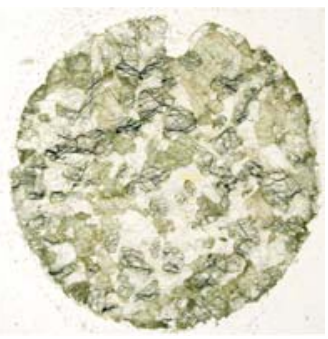

304-U1309D-268R-2, $83-85 \mathrm{~cm}, \mathrm{PPL}$

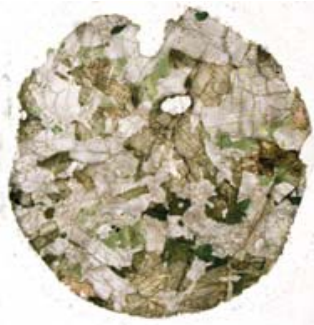

304-U1309D-275R-3, $88-90 \mathrm{~cm}, \mathrm{PPL}$

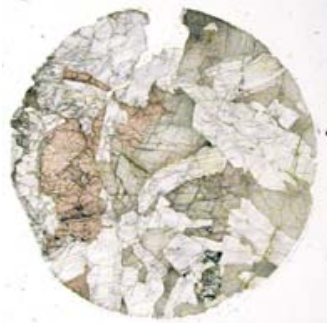

304-U1309D-285R-1, 0-92 cm, PPL

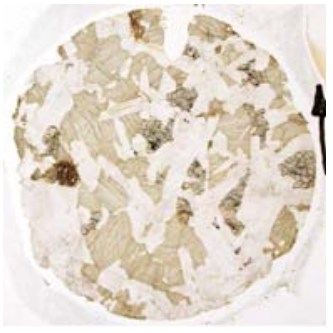

304-U1309D-291R-3, $39-41 \mathrm{~cm}, \mathrm{PPL}$ 